\title{
Control of Autophagosome Axonal Retrograde Flux by Presynaptic Activity Unveiled Using Botulinum Neurotoxin Type A
}

\author{
Tong Wang, ${ }^{1}$ CSally Martin, ${ }^{1}$ Andreas Papadopulos, ${ }^{1}$ Callista B. Harper, ${ }^{1}$ Timur A. Mavlyutov, ${ }^{4}$ Dhevahi Niranjan, ${ }^{5}$ \\ Nick R. Glass, ${ }^{2}$ Justin J. Cooper-White, ${ }^{2,3,6}$ Jean-Baptiste Sibarita, ${ }^{7,8}$ Daniel Choquet, ${ }^{7,8,9}$ Bazbek Davletov, ${ }^{10}$ \\ and Frédéric A. Meunier ${ }^{1}$ \\ ${ }^{1}$ Clem Jones Centre for Ageing Dementia Research, Queensland Brain Institute, ${ }^{2}$ Australian Institute for Bioengineering and Nanotechnology, and ${ }^{3}$ School \\ of Chemical Engineering, University of Queensland, Brisbane, Queensland 4072, Australia, ${ }^{4}$ University of Wisconsin Medical School, Madison, Wisconsin \\ 53706, ${ }^{5}$ Medical Research Council Laboratory of Molecular Biology, Cambridge CB2 0QH, United Kingdom, ${ }^{6}$ Materials Science and Engineering Division, \\ Commonwealth Scientific and Industrial Research Organization, Clayton, Victoria 3169, Australia, ${ }^{7}$ University of Bordeaux, Interdisciplinary Institute for \\ Neuroscience, ${ }^{8}$ Centre National de la Recherche Scientifique, Unité Mixte de Recherche 5297, Bordeaux, France, ${ }^{9}$ Bordeaux Imaging Center, Unité Mixte de \\ Service 3420, Centre National de la Recherche Scientifique, US4 INSERM, University of Bordeaux, France, and ${ }^{10}$ Department of Biomedical Science, The \\ University of Sheffield, Sheffield, S10 2TN, United Kingdom
}

Botulinum neurotoxin type A (BoNT/A) is a highly potent neurotoxin that elicits flaccid paralysis by enzymatic cleavage of the exocytic machinery component SNAP25 in motor nerve terminals. However, recent evidence suggests that the neurotoxic activity of BoNT/A is not restricted to the periphery, but also reaches the CNS after retrograde axonal transport. Because BoNT/A is internalized in recycling synaptic vesicles, it is unclear which compartment facilitates this transport. Using live-cell confocal and single-molecule imaging of rat hippocampal neurons cultured in microfluidic devices, we show that the activity-dependent uptake of the binding domain of the BoNT/A heavy chain (BoNT/A-Hc) is followed by a delayed increase in retrograde axonal transport of BoNT/A-Hc carriers. Consistent with a role of presynaptic activity in initiating transport of the active toxin, activity-dependent uptake of BoNT/A in the terminal led to a significant increase in SNAP25 cleavage detected in the soma chamber compared with nonstimulated neurons. Surprisingly, most endocytosed BoNT/A-Hc was incorporated into LC3-positive autophagosomes generated in the nerve terminals, which then underwent retrograde transport to the cell soma, where they fused with lysosomes both in vitro and in vivo. Blocking autophagosome formation or acidification with wortmannin or bafilomycin $A_{1}$, respectively, inhibited the activity-dependent retrograde trafficking of BoNT/A-Hc. Our data demonstrate that both the presynaptic formation of autophagosomes and the initiation of their retrograde trafficking are tightly regulated by presynaptic activity.

Key words: autophagosome; axon; Botulinum neurotoxin; presynaptic; retrograde transport; synaptic vesicle

\section{Introduction}

Botulinum neurotoxins (BoNTs) are a family of extremely potent neurotoxins comprising seven serotypes (BoNT/A-G) produced

\footnotetext{
Received Sept. 9, 2014; revised March 9, 2015; accepted March 11, 2015

Author contributions: T.W., S.M., A.P., C.B.H., T.A.M., B.D., and F.A.M. designed research; T.W., S.M., C.B.H., T.A.M., D.N., and N.R.G. performed research; C.B.H., N.R.G., J.J.C.-W., J.-B.S., and D.C. contributed unpublished reagents/analytic tools; T.W., S.M., and A.P. analyzed data; T.W., S.M., A.P., C.B.H., T.A.M., N.R.G., B.D., and F.A.M. wrote the paper.

This work was supported by project grant 1044014 and a Medical Research Council of Australia (Senior Research Fellowship 569596 to F.A.M.), the Australian Research Council (LIEF Grant LE0882864 to F.A.M.), and the University of Queensland (Postdoctoral Research Fellowship 2012001396 to T.W.). Electron microscopy was performed in the Australian Microscopy and Microanalysis Facility at the Centre for Microscopy and Microanalysis at the University of Queensland. The fabrication of the microfluidic devices was performed at the Queensland node of the Australian National Fabrication Facility, a company established under the National Collaborative Research Infrastructure Strategy to provide nanofabrication and microfabrication facilities for Australia's researchers. We thank the Bordeaux Imaging Center, part of the FranceBiolmaging national infrastructure supported by the French National Research Agency (ANR-10-INSB-04); Rowan Tweedale for critical reading of the manuscript; and Rachel Gormal, Vanesa Tomatis, Luke Hammond, Victor Anggono, and Dusan Matusica for expert technical assistance.

The authors declare no competing financial interests.
}

by different species of the genus Clostridium (Schiavo et al., 2000; Meunier et al., 2003; Rossetto et al., 2014). BoNTs are widely used in cosmetic applications and as therapeutic agents for various neurological afflictions (Foran et al., 2003; Meunier et al., 2003). The most widely used serotype is BoNT/A, which has a $50 \mathrm{kDa}$ catalytic light chain (Lc) linked to a $100 \mathrm{kDa}$ heavy chain, which has two functionally distinct domains: a binding domain $(\mathrm{Hc})$ and a translocation domain $\left(\mathrm{H}_{\mathrm{N}}\right)$ (Koriazova and Montal, 2003). BoNT/A-Hc mediates high-affinity binding to dual receptors, the ganglioside GT1b, and the protein receptor SV2C on the presynaptic plasma membrane to initiate uptake into synaptic vesicles in motor nerve terminals (Mahrhold et al., 2006; Benoit et al.,

Correspondence should be addressed to Frédéric A. Meunier Clem Jones Centre for Ageing Dementia Research, Queensland Brain Institute, The University of Queensland, Brisbane, Queensland 4072, Australia. E-mail: f.meunier@uq.edu.au.

DOI:10.1523/JNEUROSCI.3757-14.2015

Copyright $\odot 2015$ the authors $\quad 0270-6474 / 15 / 356179-16 \$ 15.00 / 0$ 
2014). Upon acidification of the vesicle lumen, BoNT/A- $\mathrm{H}_{\mathrm{N}}$ undergoes a conformational change that mediates the translocation and cytosolic release of BoNT/A-Lc, which subsequently cleaves the SNARE protein SNAP25 (Blasi et al., 1993; Schiavo et al., 1993; Rossetto et al., 2014), preventing synaptic vesicle exocytosis and causing flaccid paralysis. However, the effect of BoNT/A is not restricted to the periphery. Indeed, recent studies have uncovered central effects resulting from the retrograde axonal transport of the neurotoxin and its transfer to afferent synapses (Antonucci et al., 2008; Caleo et al., 2009; Restani et al., 2011). Furthermore, in primary motor neurons, this retrograde transport occurs together with that of tetanus toxin and the neurotrophin receptor $\mathrm{p} 75^{\mathrm{NTR}}$ (Restani et al., 2012). Importantly, the underlying cellular machinery facilitating BoNT/A retrograde flux is still largely unknown.

Macroautophagy, usually referred to as autophagy, is a major system for the degradation of long-lived proteins and organelles, and the retrograde autophagosome pathway plays critical roles in maintaining functional homeostasis and survival in neurons (Wang et al., 2006; Xie and Klionsky, 2007; Katsumata et al., 2010; Maday and Holzbaur, 2012a, 2012b; Shehata et al., 2012; Martin et al., 2013). Autophagosome biogenesis occurs constitutively in presynaptic nerve terminals and autophagosomes undergo dynein-dependent retrograde axonal transport to the neuronal soma, where they fuse with lysosomes (Xie and Klionsky, 2007; Maday and Holzbaur, 2012b). Because the biogenesis of autophagosomes occurs concurrently with synaptic vesicle recycling in nerve terminals (Katsumata et al., 2010; Shehata et al., 2012), we explored whether stimulation could affect the generation of this specialized pool of autophagosomes. Given that BoNT/A-Hc is internalized in synaptic vesicles (Harper et al., 2011) and then undergoes retrograde trafficking (Restani et al., 2012), we used BoNT/A-Hc as a specialized synaptic vesicle cargo to investigate the interrelationship between autophagosome formation and retrograde synaptic component trafficking. We reveal that a substantial proportion of BoNT/A-Hc undergoes retrograde transport within autophagosomes and that the retrograde flux of both BoNT/A-Hc and autophagosomes is highly dependent on the level of presynaptic activity. Our data demonstrate that a transient increase in presynaptic activity upregulates presynaptic autophagy and suggest a molecular link between presynaptic activity and presynaptic autophagosome biogenesis.

\section{Materials and Methods}

Animals. For in vivo experiments, adult male C57BL/6 mice were used. For hippocampal cultures, female Sprague Dawley rat dams were killed and brain tissue was from embryos of both sexes. All experiments were approved by the Animal Ethics Committee at the University of Queensland and were conducted according to the Australian Code of Practice for the Care and Use of Animals for Scientific Purposes.

Antibodies and reagents. Antibodies were obtained from the following sources: rabbit anti-LC3 (Novus Biologicals, catalog \#NB100-2331; Cell Signaling Technology, catalog \#3868), mouse anti- $\beta$-actin (Sigma, cata$\log$ \#S0644), mouse anti $\beta$ III-tubulin (Covance, catalog \#MMS-435P), and rabbit anti-LAMP1 (Sigma, catalog \#L1418; Abcam, catalog \#ab24170). pEGFP-LC3 (plasmid 21073; Kabeya et al., 2000) and pmRFP-LC3 (plasmid 21075; Kimura et al., 2007) were generated in the laboratory of Tamotsu Yoshimori (Osaka University, Japan) and obtained from Addgene. The BoNT/A-Lc was subcloned into pEGFP-N1 to make pEGFP-BoNT/A-Lc from the pCMV-BoNT/A-Lc construct (a gift from Thomas Binz, Institut für Biochemie, Medizinische Hochschule Hannover, Hannover, Germany), FITC-conjugated p $75^{\text {NTR }}$ monoclonal antibody (Matusica et al., 2013) was a gift from Elizabeth Coulson (Queensland Brain Institute, University of Queensland, Brisbane, Australia). BoNT/A-truncated SNAP25 antibody was a kind gift from D.
Sesardic (Division of Bacteriology, National Institute for Biological Standards and Control, Hertfordshire, United Kingdom). LysoTracker Red DND-99 was from Thermo Scientific (catalog \#L-7528). Fluorescently conjugated secondary antibodies were obtained from Invitrogen. The remaining reagents were sourced from Sigma unless stated otherwise.

BoNT/A-Hc expression and conjugation. His6-tagged BoNT/A-Hc was purified using Co IDA-agarose (Scientifix) as described previously (Couesnon et al., 2009; Harper et al., 2011), then conjugated to Atto647N maleimide (Atto-TEC, catalog \#647N-41) according to the manufacturer's instructions. For BoNT/A-Hc-HRP conjugation, the EZ-Link Maleimide Activated Horseradish Peroxidase Kit (Thermo Scientific, catalog \#31494) was used to conjugate HRP to sulfhydryl groups of BoNT/A-Hc. A 1:2 molar ratio of BoNT/A-Hc and activated HRP were incubated in conjugation buffer at $4^{\circ} \mathrm{C}$ overnight. The conjugated BoNT/ A-Hc-HRP was purified using a PD MiniTrap G-25 Column (GE Healthcare, catalog \#28-9180-08) following the gravity protocol. The protein concentration was measured using the Bradford reagent (Bio-Rad, cata$\log \# 500-0006)$. All conjugated proteins were stored at $-80^{\circ} \mathrm{C}$ with the addition of $0.1 \%$ bovine serum albumin (BSA) as a stabilizer.

Neuronal cultures. Rat hippocampal neurons were prepared as described previously (Wang et al., 2011) and seeded in microfluidic devices (Xona, catalog \#RD450) following the manufacturer's protocol (Taylor et al., 2005). For the single-molecule tracking experiments, devices were design based on aforementioned devices but adapted to fit on $22 \mathrm{~mm}$, glass-bottomed, super-resolution compatible culture dish dishes (In Vitro Scientific, catalog \#D29-20-1.5-N). Axon extension device molds were made through standard multilayer SU8 photolithographic means and devices were molded using standard soft lithographic techniques. Devices and culture dishes were then exposed to oxygen plasma (Harrick) and bonded. Freshly bonded devices were baked at $65^{\circ} \mathrm{C}$ overnight to facilitate bonding. Immediately before use, the devices were exposed to oxygen plasma to increase the wettability of the device. Afterward, in a sterile environment, the devices were filled and rinsed with $70 \%$ ethanol and Milli-Q water (Millipore), after which they were coated for $1 \mathrm{~h}$ with poly-D-lysine at $0.1 \%$ at $37^{\circ} \mathrm{C}$. The devices were subsequently rinsed with Milli-Q water, PBS, and plating medium. The devices were left in plating medium until neurons were seeded. The devices were seeded by adding a highly concentrated suspension of cells $\left(10^{7}\right.$ cells $\left./ \mathrm{ml}\right)$ to the soma chamber only. The cells were then allowed to attach for $1 \mathrm{~h}$ before the medium reservoirs were filled with plating medium. Cultured neurons were transfected using Lipofectamine LTX with Plus Reagent (Thermo Scientific, catalog \#15338100) after $12 \mathrm{~d}$ in vitro (DIV 12). Live-imaging experiments were performed $48 \mathrm{~h}$ after transfection.

Stimulation and nerve terminal labeling with BoNT/A-Hc: fluorescence microscopy. Stimulation and labeling were performed on rat hippocampal neurons cultured in microfluidic devices between DIV 14 and 17. Briefly, the culture medium was removed from all chambers and neurons were incubated for $5 \mathrm{~min}$ at $37^{\circ} \mathrm{C}$ in low $\mathrm{K}^{+}$buffer containing the following (in mM): $15 \mathrm{HEPES}, 145 \mathrm{NaCl}, 5.6 \mathrm{KCl}, 2.2 \mathrm{CaCl}_{2}, 0.5 \mathrm{MgCl}_{2}, 5.6$ D-glucose, and 0.5 ascorbic acid plus $0.1 \%$ BSA, pH 7.4, or high $\mathrm{K}^{+}$ buffer containing the same ingredients as the low $\mathrm{K}^{+}$buffer except that it contained $95 \mathrm{~mm} \mathrm{NaCl}$ and $56 \mathrm{~mm} \mathrm{KCl}$ (Fu et al., 2009; Harper et al., 2011; Martin et al., 2013), with $100 \mathrm{~nm}$ BoNT/A-Hc-Atto647N added to the nerve terminal chambers only. For the single-particle tracking experiments, $1 \mathrm{~nm}$ BoNT/A-Hc-Atto647N was used. Devices were then returned to the original medium for $2 \mathrm{~h}$ before imaging. Images were acquired with a Zeiss LSM710 inverted microscope maintained at $37^{\circ} \mathrm{C}$ and $5 \% \mathrm{CO}_{2}$ and videos were analyzed for carrier kinetics using the spot function of Imaris software (Imaris version 7.7; Bitplane). Kymographs were generated using ImageJ software using the plugin MultiKymograph. For immunofluorescence microscopy of fixed cells, microfluidic devices were removed and neurons were fixed at various time points with PBS containing 4\% paraformaldehyde and 4\% sucrose and then processed for immunocytochemistry as described previously (Wen et al., 2008). Permeabilization was performed using $0.1 \%$ saponin, $0.2 \%$ gelatin, and 1\% BSA in PBS. Imaging was performed on a Zeiss LSM 710 confocal microscope and analyzed with Zen (Zeiss) and ImageJ software. All images were processed using Adobe Photoshop CS 5.1 and figures were compiled using Adobe Illustrator CS 5.1. 
Electron microscopy. Rat hippocampal neurons cultured in microfluidic devices were stimulated as described above and then incubated for a further 3-4 h, after which the cells were washed in PBS, the microfluidic device removed, and the cells fixed in 2.5\% glutaraldehyde in PBS. To measure BoNT/A-Hc-HRP trafficking, rat hippocampal neurons cultured in microfluidic devices were treated as described for confocal microscopy except that $100 \mathrm{~nm}$ BoNT/A-Hc-HRP was added to the nerve terminal chambers for the period of stimulation. Cells were returned to growth medium for $3 \mathrm{~h}$ before fixation. After fixation, cells were processed for 3,39-diaminobenzidine (DAB) cytochemistry using standard protocols. Fixed cells were contrasted with $1 \%$ osmium tetroxide and $4 \%$ uranyl acetate before dehydration and embedding in LX-112 resin (Harper et al., 2011). Ultrathin sections $(\sim 60 \mathrm{~nm})$ were cut using an ultramicrotome (UC64; Leica). Sections were visualized on a transmission electron microscope (model 1011; JEOL) equipped with a cooled charge-coupled device camera (Morada; Olympus). Autophagosomes within the cell soma proximal region of the microfluidic channel were counted and scored for the presence or absence of the DAB reaction product using Image software.

$p 75^{N T R}$ and LysoTracker labeling and colocalization analysis. DIV 14 hippocampal neurons cultured in microfluidic devices were incubated at $37^{\circ} \mathrm{C}$ with $50 \mathrm{~nm}$ LysoTracker for $30 \mathrm{~min}$ or with an antibody against the extracellular domain of $\mathrm{p} 75^{\mathrm{NTR}}$ (1/500 diluted) for $1 \mathrm{~h}$ (Matusica et al., 2013). After incubation, neurons were pulse-chase labeled with fluorescently labeled BoNT/A-Hc under high $\mathrm{K}^{+}$or low $\mathrm{K}^{+}$conditions as described above and then washed with culture medium before being imaged with an iLas ${ }^{2}$ microscope (Roper Scientific) fitted with a $100 \times$ oil-immersion objective (numerical aperture 1.49; Nikon) and a highperformance EMCCD camera (Photometrics, Evolve 512 Delta) with MetaMorph software. Axons were visualized in microgrooves using liveimaging buffer containing the following (in mM): 25 HEPES, $145 \mathrm{NaCl}$, $5.6 \mathrm{KCl}, 0.5 \mathrm{MgCl}_{2}, 2.2 \mathrm{CaCl}_{2}$, and 5.6 D-glucose, $\mathrm{pH}$ 7.4. Time-lapse images of two fluorophores were captured at $0.1-0.3 \mathrm{~s}$ intervals for $95 \mathrm{~s}$. Carriers were tracked automatically with the "surface" function of Imaris software (Imaris version 7.7; Bitplane) and the same parameter settings were used in both the low $\mathrm{K}^{+}$- and high $\mathrm{K}^{+}$- treated groups. The colocalization ratio between different channels was calculated with the "colocalization" function of Imaris software, with the ratio of all time points accumulated to generate the total ratio within a movie. Statistical analyses and bar graphs were generated using Prism 6 (GraphPad Software).

In vivo injection of mCherry-BoNT/A-Hc. BoNT/A-Hc fused to mCherry was generated in pGEX-KG by introducing the mCherry sequence into the vector, followed by the BoNT/A-Hc sequence (aa 8731296, NCBI sequence P10845; Binz et al., 1990). The mCherry-BoNT/ A-Hc protein was expressed in the BL21 strain of E. coli as a GST C-terminal fusion protein before being purified on glutathione Sepharose beads (GE Healthcare) and eluted using thrombin. Further purification was performed by gel filtration using a Superdex 200 10/200 GL column (GE Healthcare). For in vivo experiments, male C57BL/6 mice were anesthetized with isoflurane, followed by intraplantar injections of mCherry-BoNT/A-Hc $(1 \mu \mathrm{g})$ into the right foot. After $24 \mathrm{~h}$, the mice were killed and slices were prepared from the L4-S2 region of the spinal cord. Slices containing a red fluorescent signal in the lateral column were selected under a dissecting fluorescent microscope (Nikon, SMZ1500) and labeled for immunofluorescence microscopy using LC3 or LAMP1 antibodies. Images were acquired on an A1R laser confocal microscope (Nikon) and analyzed with NIS elements software.

Single-particle detection of internalized BoNT/A-Hc in hippocampal axons. Transfected neurons cultured in microfluidic devices on glassbottomed culture dishes (In Vitro Scientific, catalog \#D29-20-1.5-N) were stimulated with high $\mathrm{K}^{+}$buffer for 5 min with $1 \mathrm{nM}$ BoNT/A-HcAtto647N added to the nerve terminal chamber only, and then washed 3 times with culture medium and chased for $2-4 \mathrm{~h}$ in a $37^{\circ} \mathrm{C}$ incubator. Axon channels were visualized in live-imaging buffer at $37^{\circ} \mathrm{C}$. Cells were acquired on a motorized inverted microscope (Nikon, TiE) equipped with a perfect focus system under slightly oblique illumination (ILas ${ }^{2}$; Roper Scientific) using a $100 \times 1.49$ numerical aperture CFI Apo objective (Nikon). Time-lapse movies were captured at $20 \mathrm{frames} / \mathrm{s}$ through an EM-CCD (Evolve512 delta; Photometrics) using MetaMorph software
(Molecular Devices) for the indicated period and analyzed using custommade single-molecule analysis software (Kechkar et al., 2013; Nair et al., 2013). To isolate trajectories of carriers that underwent directed motion from nonspecific noise and immobile objects, we selected the trajectories based on their duration ( $>25$ consecutive frames) and with a mean square displacement (MSD) (Steyer and Almers, 1999; Papadopulos et al., 2013) fitting a polynomial equation of the type: $\left\langle r^{2}\right\rangle(t)=a t^{2}+b$ with $R^{2}>0.99$ (Maucort et al., 2014). Using this fit, we also extracted trajectory speeds using $\sqrt{a}=v$. The spatial resolution $(73 \pm 5 \mathrm{~nm})$ of our experiments was extracted by interpolating the MSD.

Statistic analysis. After analysis for the normal distribution and homogeneity of variance among values of the same set, these data were subjected to statistical analysis using one-way ANOVA supplemented with $t$ tests. Unless otherwise noted, sample number $(n)$ represents the number of axon channels for video acquisition and each experiment was repeated at least three times. All data are shown as mean \pm SEM, with $p<0.05$ considered to be a significant difference.

\section{Results}

\section{Retrograde trafficking of BoNT/A-Hc in hippocampal neurons is increased by presynaptic activity}

BoNT/A enters nerve terminals by an activity-dependent process (Dolly et al., 1984; Harper et al., 2011). Recent studies have shown that the toxin can subsequently undergo retrograde axonal transport, leading to previously unreported pathological consequences (Verderio et al., 2007; Antonucci et al., 2008; Restani et al., 2012). However, the identity of the retrograde carriers transporting the toxin back to the cell soma remains to be fully elucidated. To address this question, we used primary hippocampal neurons cultured in microfluidic devices to spatially separate axon nerve terminals from the somato-dendritic area (Fig. $1 A, B)$. To avoid toxin reaching the soma chamber through the axon channels during the 5 min pulse-labeling process, we induced a hydrostatic pressure difference between the two chambers separated by the microgrooves, driving a microfluidic flow from the soma to the terminal chamber, thereby restricting the toxin in the terminal chamber (Taylor et al., 2005). BoNT/A endocytosis and trafficking were investigated by pulsing the nerve terminal chamber with an Atto647-conjugated binding domain of the BoNT/A-Hc (100 nM) for 5 min in either a high $\mathrm{K}^{+}$buffer to induce synaptic vesicle recycling or in a control low $\mathrm{K}^{+}$buffer. This was followed by a thorough wash in culture medium to remove the excess probe and confocal time-lapse imaging. To maximize our analysis of retrograde carriers, imaging and quantification were performed in the axon channels immediately adjacent to the soma chamber. Although retrograde carriers were very rare in the first hour of analysis in both experimental conditions, a clear increase in the flux of BoNT/A-Hc retrograde carriers occurred from 2 to $4 \mathrm{~h}$ (Fig. 1C). This was particularly evident in cells that had been pulse labeled in a depolarizing high $\mathrm{K}^{+}$buffer (Fig. 1C). We found an $\sim 3$-fold increase in the number of retrograde BoNT/A-Hc-positive carriers in neurons treated with a single $5 \mathrm{~min}$ pulse of high $\mathrm{K}^{+}(2.0 \pm 0.3 / \mathrm{min})$ compared with low $\mathrm{K}^{+}$-treated cells $(0.7 \pm 0.1 / \mathrm{min}$; Fig. $1 D)$. We could not detect any difference in the number of stationary or anterograde BoNT/A-Hc-positive carriers (Fig. 1D). We subsequently analyzed the kinetic parameters of these carriers and found that stimulation did not affect the average speed of retrograde, stationary or anterograde BoNT/A-Hc-positive carriers (Fig. 1E). Indeed, the patterns of the tracks were similar between the low $\mathrm{K}^{+}$and high $\mathrm{K}^{+}$groups, as seen in the kymographs (Fig. $2 A$ ), although the flux of carriers was clearly increased in the stimulated group. This was confirmed by frequency distribution analysis of the average speeds, which showed the similar peaks 
between the two groups (Fig. 2B), and analysis of the instant speed of BoNT/AHc-positive carriers, which was also unaffected by stimulation (Fig. 2C). Finally, we analyzed the "dwelling time," defined as carriers with an instant speed of $<0.7$ $\mu \mathrm{m} / \mathrm{s}$, expressed as a percentage of total time spent in the imaging area. This revealed no change in the dwelling time (Fig. 2D). These data demonstrate that raising presynaptic activity selectively increases the number of BoNT/A-Hcpositive carriers undergoing retrograde axonal transport without affecting other dynamic parameters such as speed and dwelling time. This suggests that the level of presynaptic activity influences the generation of BoNT/A-Hc-positive retrograde carriers, but does not affect the underlying mechanism driving these carriers with specific molecular motors.

Because we used a higher concentration of BoNT/A-Hc (100 nM) than that of BoNT/A holotoxin (Harper et al., 2011), which has been reported to generate pathological conditions (Pellizzari et al., 1999), we considered the possibility that this may affect the binding specificity and subsequent sorting to recycling vesicles. We therefore also examined the fate of internalized BoNT/A-Hc at lower, more pathologically relevant concentrations. Conventional confocal microscopy is unable to detect such a low concentration of endocytosed BoNT/A-Hc-Atto647N because only a few molecules are likely to enter each retrograde carrier. We therefore adopted the use of a more sensitive, singlemolecule detection technique. BoNT/AHc-Atto647N (1 nM) was internalized in the nerve terminals of neurons cultured in microfluidic devices under high $\mathrm{K}^{+}$ conditions and chased for 2-4 h (Fig. 3A). The hallmark of single-molecule detection is the stochastic disappearance of fluorescence resulting from photobleaching. Using superresolution microscopy, we were able to detect single molecules of internalized BoNT/A-Hc-Atto647N traveling in the axon channels (Fig. $3 B)$. The trajectories of single molecules of BoNT/A-Hc were traced and analyzed with MetaMorph PALM tracer (Nair et al., 2013). Importantly, we could detect molecules undergoing retrograde-directed motion within the axons (Fig. $3 B$ ). Because we predicted that these would be transported within retrograde carriers, we screened for directed motion trajectories using a mathematical filter based on fitting trajectory MSDs to a polynomial equation (see Materials and Methods), fitting curves as shown in Figure $3 C$. This allowed us to calculate the speed of each transported molecule of BoNT/A-Hc and compare it with that of BoNT/A-Hc carriers measured using conventional confocal microscopy (Fig. 3D). Importantly, we found that the average speed of the single BoNT/A-Hc molecules undergoing axonal retrograde movement was not significantly different from that obtained using the higher concentration of BoNT/A-Hc and imaged $t$ test.
$5 \mathrm{~min}$ pulse
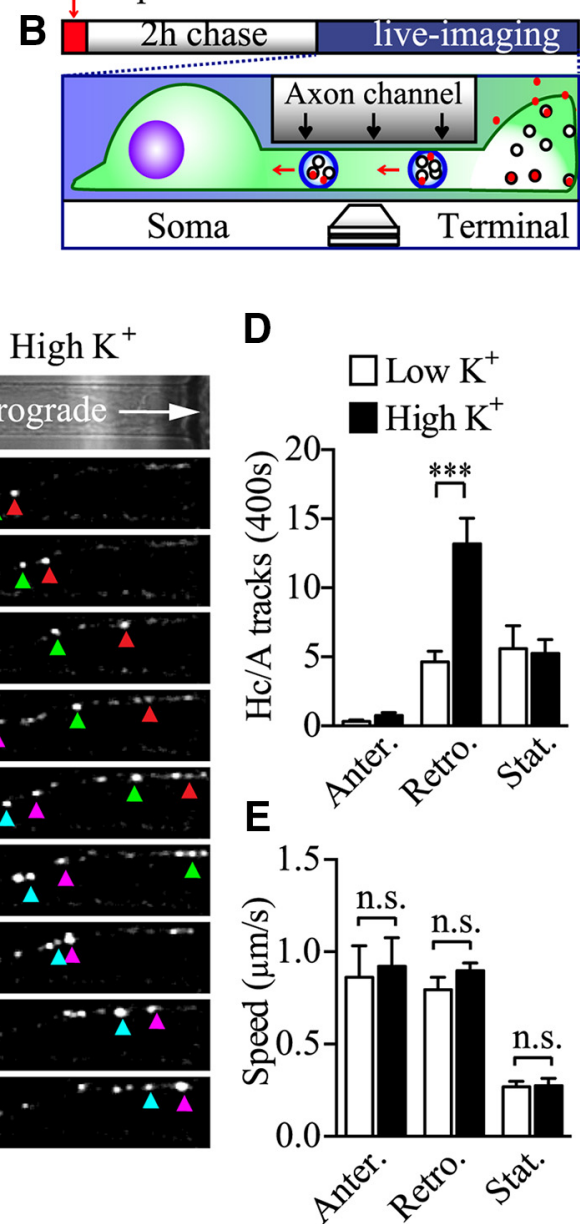

Figure 1. Axonal retrograde trafficking of BoNT/A-Hc-positive carriers is increased by activity. $A$, Photograph of the microfluidic devices used to compartmentalize nerve terminals from the somatodendritic area. Scale bar, $1 \mathrm{~cm}$. $\boldsymbol{B}$, Scheme illustrating the hamber in the presence of low or high $\mathrm{K}^{+}$buffer, washed in culture medium, and imaged by time-lapse confocal microscopy from $(\boldsymbol{D})$ and average speed $(\boldsymbol{E})$ were calculated in the indicated experimental conditions. Data from 5 independent neuron preparations are shown; $n=34$ and 31 individual channels for low $\mathrm{K}^{+}$and high $\mathrm{K}^{+}$, respectively. ${ }^{* *} p<0.001 ; n$.S., not significant, Student's

by time-lapse confocal microscopy (Fig. 3D), strongly suggesting that these were indeed the same carriers. Our results confirm that BoNT/A-Hc, in the range of concentrations used in this study, specifically enters an endocytic compartment that ultimately leads to the generation of fast axonal retrograde carriers.

\section{Retrograde trafficking of BoNT/A holotoxin is regulated by presynaptic activity}

Because the light chain of BoNT/A (Lc/A) is the protease that specifically cleaves the C terminus of SNAP25, thereby blocking neurotransmitter release (Pellizzari et al., 1999; Schiavo et al., 2000; Meunier et al., 2002), exogenously expressed GFP-tagged BoNT/A-Lc/A (GFP-Lc/A) can be used to specifically block neuroexocytosis in transfected neurons (Bajohrs et al., 2004). To confirm that BoNT/A-Hc uptake and retrograde transport are dependent on the level of presynaptic activity, hippocampal neurons transfected with GFP-Lc/A were subjected to BoNT/A-Hc pulse labeling under high $\mathrm{K}^{+}$stimulation and the amount of BoNT/A-Hc in the GFP-Lc/A-transfected neurons was compared 

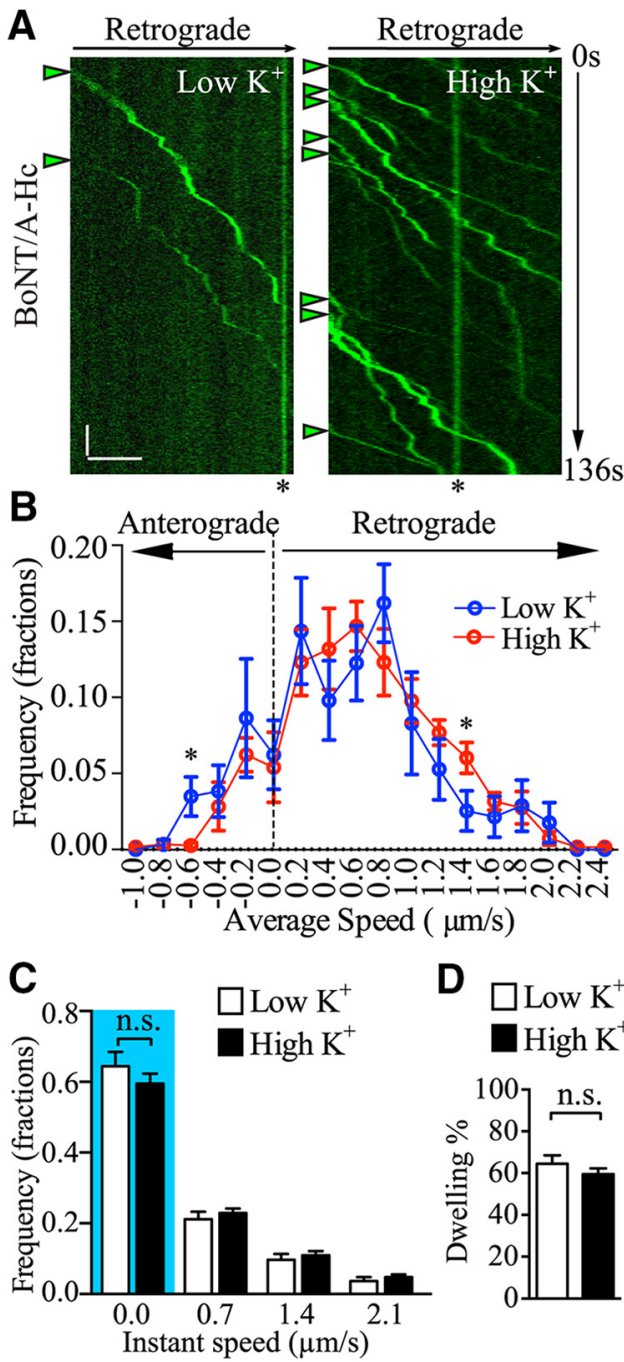

D $\square$ Low K $\mathrm{K}^{+}$ High $\mathrm{K}^{+}$

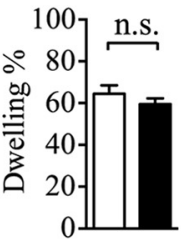

Figure 2. The kinetics of retrograde BoNT/A-Hc carriers is not affected by presynaptic activity. $\boldsymbol{A}$, Representative kymographs of BoNT/A-Hc carriers generated by a line scan following a single axon track on the time-lapse image stacks. Arrowheads indicate retrograde carriers and asterisks stationary compartments. Scale bars: $x$-axis, $10 \mu \mathrm{m}$; $y$-axis, 10 s. $\boldsymbol{B}, \boldsymbol{C}$, Frequency distribution of average speed $(\boldsymbol{B})$ and instant speed $(\boldsymbol{C})$ of BoNT/A-Hc carriers after a 5 min pulse in either low $\mathrm{K}^{+}$or high $\mathrm{K}^{+}$treatment followed by a 2-4 $\mathrm{h}$ chase. Dwelling time defined by tracks times with an instant speed of $0-0.7 \mu \mathrm{m} / \mathrm{s}$ is quantified in $\boldsymbol{C}$ (blue highlight) and $\boldsymbol{D}$ as percentage dwelling time relative to the total track time shown in the indicated conditions. Data from 5 independent neuron preparations are shown; $n=34$ and 31 individual channels for low $\mathrm{K}^{+}$and high $\mathrm{K}^{+}$, respectively. ${ }^{*} p<0.05$; n.S., not significant, Student's $t$ test.

with that of adjacent nontransfected neurons (Fig. 4A). In addition, the retrograde transport of BoNT/A-Hc to the soma after a $2-4 \mathrm{~h}$ chase was also compared (Fig. $4 B$ ). As expected, we found significantly less BoNT/A-Hc (Hc/A) uptake in the nerve terminals of GFP-Lc/A-transfected neurons compared with the adjacent nontransfected neurons (Fig. $4 A$, top right). The enzymatic cleavage activity of exogenous expressed GFP-Lc/A in these cells was confirmed by immunofluorescence using an antibody specifically designed to recognize BoNT/A-cleaved SNAP25 (SNAP25A; Ekong et al., 1997; Foran et al., 2003; Fig. 4A, right middle and bottom). Similarly, the retrograde transport of BoNT/A-Hc to the neuronal soma after pulse-chase labeling was also significantly decreased in GFP-Lc/A-expressing neurons (Fig. 4B). Quantification of these data is shown in Figure 4, $C$ and $D$. These results confirm that the uptake of BoNT/A-Hc is dependent on presynaptic activity in hippocampal neurons (Dong et al., 2006; Harper et al., 2011; Colasante et al., 2013) and reveal that subsequent BoNT/A-Hc fast axonal retrograde transport is also upregulated by activity.

Although the binding domain of BoNT/A is a useful probe with which to visualize the endocytosis and retrograde transport of this toxin, it is unable to recapitulate the complete intoxication process that occurs in neurons. Therefore, we determined whether retrogradely transported BoNT/A holotoxin remained active upon reaching the soma compartment and if it was affected by raising presynaptic activity. DIV 14 hippocampal neurons cultured in microfluidic devices were subjected to a 5 min incubation with BoNT/A holotoxin (100 pM) in the nerve terminal chamber under high $\mathrm{K}^{+}$or low $\mathrm{K}^{+}$conditions, and a hydrostatic pressure difference between the 2 chambers to fluidically isolate each chamber was maintained during the pulse (Taylor et al., 2005). After extensive washes and a $24 \mathrm{~h}$ chase, neurons were fixed and immunostained for SNAP25A (Fig. 4E). Consistent with a previous report (Restani et al., 2012), SNAP25A labeling was detected in both the soma and terminal chambers in both stimulated and unstimulated neurons. Interestingly, image analysis revealed a stronger SNAP25A signal in both the terminal (Fig. 4E, F) and soma (Fig. $4 E, G$ ) chambers after high $\mathrm{K}^{+}$stimulation. The specificity of the SNAP25A was confirmed by the low signal detected in the chamber in the absence of BoNT/A (Fig. 4E).

The immunolabeling results were confirmed by Western blotting lysates derived from the somatic chamber of high-density neuronal cultures for SNAP25A (Fig. 4H). To verify the specificity of the SNAP25A antibody, we included a positive control (BoNT/A was directly added to the somatic chamber itself) and a negative control (no toxin was added to any chamber) in addition to the high $\mathrm{K}^{+}$-stimulated and -unstimulated groups. Probing the intoxicated soma chamber from stimulated neurons (positive control) revealed strong protease activity, as shown in Figure $4 \mathrm{H}$ (lane 1), which was absent from the negative control (Fig. $4 \mathrm{H}$, lane 2). Upon intoxication of the terminal chamber and probing of the soma chamber, we detected clear cleavage activity (Fig. $4 \mathrm{H}$, lane 3), indicating that the holotoxin transported by axonal retrograde trafficking was still highly active upon reaching the soma chamber. Interestingly, there was a significant increase in the level of SNAP25 cleavage detected in the soma of stimulated neurons compared with unstimulated neurons (Fig. 4H, lane 4, I).

Our results are consistent with a previous study in motor neurons (Restani et al., 2012) showing that BoNT/A holotoxin endocytosed at the nerve terminal can be retrogradely transported to the soma and remain catalytically active. Interestingly, we also demonstrate that presynaptic activity up-regulates the delivery of active BoNT/A to the soma chamber.

Presynaptic activity regulates BoNT/A-Hc colocalization with p75 ${ }^{\text {NTR }}$ and acidic carriers

Having shown that retrograde transport of BoNT/A-Hc is upregulated by presynaptic activity, we next attempted to characterize the nature of the retrograde BoNT/A-Hc carriers. One important group of retrograde compartments is the signaling endosome containing the neurotrophin receptor $\mathrm{p} 75^{\mathrm{NTR}}$ (Bronfman et al., 2003). In cultured motor neurons, neurotoxins such as tetanus toxin, BoNT/A, and BoNT/E hijack this route to reach the soma of motor neurons and their carriers also contain p75 ${ }^{\text {NTR }}$ (Lalli and Schiavo, 2002; Restani et al., 2012). To determine whether retrograde BoNT/A-Hc carriers use a similar route in hippocampal neurons, we used an FITC-conjugated antibody that recognizes the rat $\mathrm{p} 75^{\mathrm{NTR}}$ extracellular domain (Matusica et al., 2013) to label the surface $\mathrm{p} 75^{\mathrm{NTR}}$ of neurons cultured in 
microfluidic devices. The labeled neurons were subjected to $5 \mathrm{~min}$ of BoNT/A-HcAtto647 pulse labeling at their nerve terminals under high $\mathrm{K}^{+}$or low $\mathrm{K}^{+}$conditions, and then washed and chased at $37^{\circ} \mathrm{C}$ for $2-4 \mathrm{~h}$. The dual fluorophores were subsequently imaged sequentially at $0.3 \mathrm{~s}$ intervals with a high-sensitivity EMCCD for $95 \mathrm{~s}$ and videos were analyzed for colocalization. In unstimulated neurons, $20.5 \pm 2.7 \%$ of BoNT/A-Hc carriers labeled for $\mathrm{p} 75^{\mathrm{NTR}}$ (Fig. 5A-C). This colocalization ratio was not significantly altered by high $\mathrm{K}^{+}$stimulation, with $17.9 \pm$ $3.0 \%$ of BoNT/A-Hc carriers also labeling for $\mathrm{p} 75^{\mathrm{NTR}}$ (Fig. $5 A-C$ ). These data indicate that some BoNT/A-Hc carriers are indeed transported to the soma via the same endosomal pathway as $\mathrm{p} 75^{\mathrm{NTR}}$. However, we could not detect an effect of increased presynaptic activity on this subset of retrograde BoNT/A-Hc carriers (Fig. 5C).

Next, we determined the potential overlap between BoNT/A-Hc carriers and acidified compartments labeled with LysoTracker (Lewis and Lentz, 1998). We incubated neurons cultured in microfluidic devices with the acidic vesicle marker LysoTracker Red before BoNT/A-Hc pulse-chase labeling and examined the colocalization ratio of LysoTracker and BoNT/A-Hc. In unstimulated neurons, a low proportion of BoNT/A-Hc carriers were LysoTracker positive $(9.1 \pm 1.8 \%$; Fig. 5D-F), consistent with the results of a previous study (Restani et al., 2012). Interestingly, we found a significantly higher overlap between BoNT/A-Hc and acidified compartments after high $\mathrm{K}^{+}$stimulation $(22.3 \pm 2.7 \%$; Fig. $5 D-$ $F)$, indicating that, in hippocampal neurons, more BoNT/A-Hc is transported through acidified compartments after increased presynaptic activity.

\section{Synaptic activity upregulates autophagosome biogenesis in nerve terminals}

To further understand the nature of the BoNT/A-Hc-positive retrograde carriers, we examined axons morphologically within the microfluidic channels using transmission electron microscopy. Direct comparison of the axons 3-4 h after stimulation revealed a striking increase in the number of autophagosomes detected in stimulated neurons (Fig. $6 A, B$ ). In contrast, the density of other organelles such as mitochondria was not significantly affected by stimulation (Fig. 6C). These results suggest that presynaptic activity upregulates autophagosome formation and retrograde transport. Next, we used BoNT/AHc-HRP (100 nM) to pulse label nerve terminals of neurons cultured in microfluidic devices for $5 \mathrm{~min}$ and then examined the presence of BoNT/A-Hc-HRP in the autophagosome compartments in axon channels using DAB cytochemistry. Significantly more electron-dense autophagosome structures were detected in the BoNT/A-Hc-HRP-labeled group compared with the unlabeled control group (Fig. $6 D, E$ ). This not only confirmed the specificity of the probe labeling, but, more im-
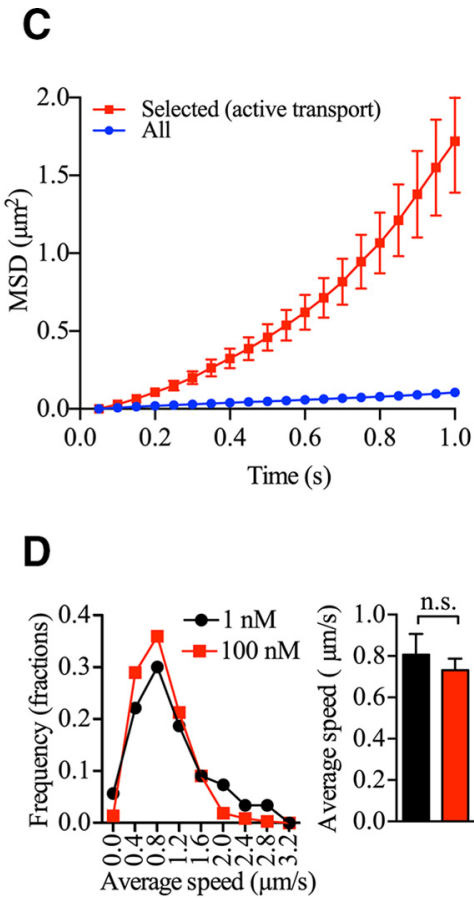

BoNT/A-Hc single molecules also undergo fast retrograde axonal transport. $\boldsymbol{A}$, Scheme illustrating BoNT/A-Hc (red axonal retrograde transport in microfluidic devices and the single-molecule detection technique used. Hippocampal neurons molecule imaging) and fluorescent carriers detected by confocal microscopy. For the $1 \mathrm{~nm}$ group, $n=21$ axon channels (a total of 176 trajectories from 3 independent neuron preparations). For the $100 \mathrm{~nm}$ group, $n=27$ axon channels (a total of 571 trajectories from 5 independent neuron preparations). n.s., Not significant, Student's $t$ test.

portantly, also provides direct evidence that nerve-terminalderived BoNT/A-Hc is sorted into autophagosomes in hippocampal neurons.

We then investigated the site of autophagosome biogenesis by analyzing the distribution of endogenous microtubuleassociated protein 1A/1B-LC 3 (LC3, an autophagosome marker) carriers between the nerve terminals and the cell soma at different times after stimulation. Neurons cultured in microfluidic chambers were stimulated with high $\mathrm{K}^{+}$for $5 \mathrm{~min}$ and then chased for $10 \mathrm{~min}$ or $120 \mathrm{~min}$ in original conditioned medium. Neurons were subsequently labeled for LC3 and $\beta$ III-tubulin. Consistent with previous studies, the basal number of LC3-positive compartments was low in both the soma and nerve terminals in unstimulated neurons (Komatsu et al., 2005; Katsumata et al., 2010; Shehata et al., 2012; Martin et al., 2013; Fig. 6F). After stimulation and incubation for $10 \mathrm{~min}$, a significant increase in LC3-positive compartments was observed in the nerve terminal chamber (Fig. $6 F, G$ ). After 120 min, a significant increase in LC3-positive compartments was observed in both the soma and the nerve terminal (Fig. $6 F$ ). Notably, after $10 \mathrm{~min}$, when LC3-positive compartments were already greatly increased in the nerve terminals, the LC3 compartments in the neuronal soma remained at a low level that was not significantly different from that of unstimulated controls (Fig. 6G,H).

To measure the effect of presynaptic activity on autophagy independently, we analyzed the level and modification of LC3 
A

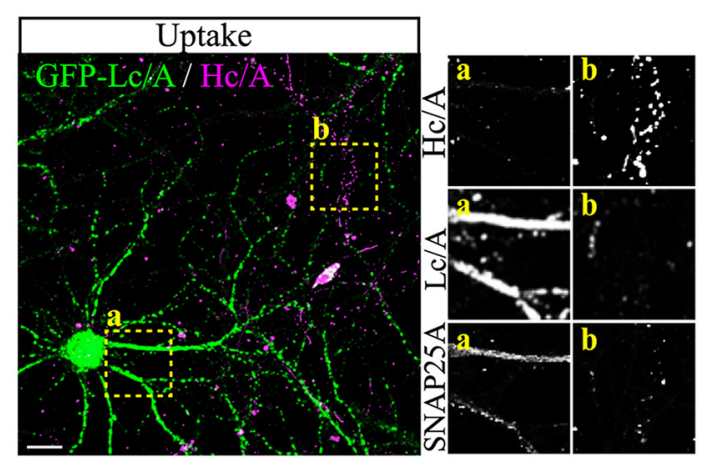

B

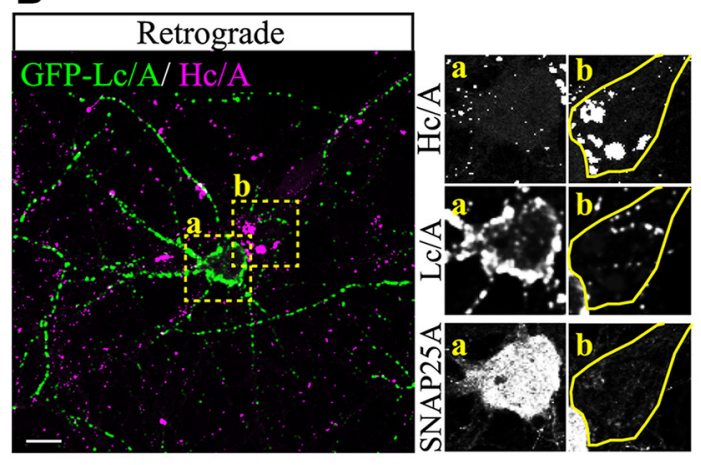

E
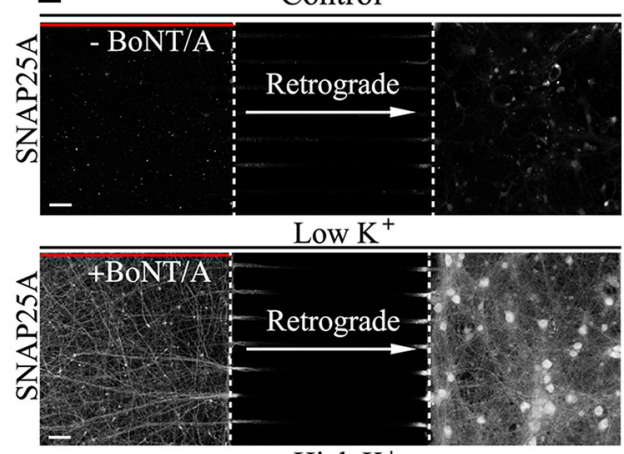

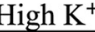

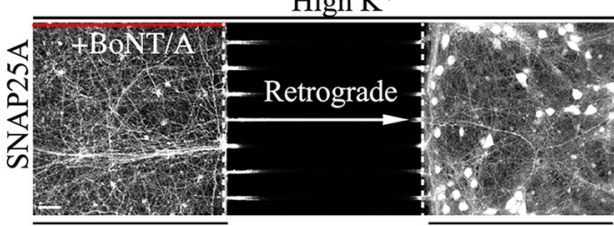

Terminal chamber

Soma chamber

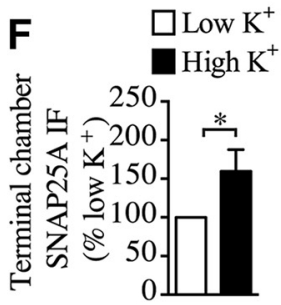

G

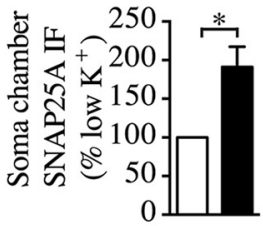

I
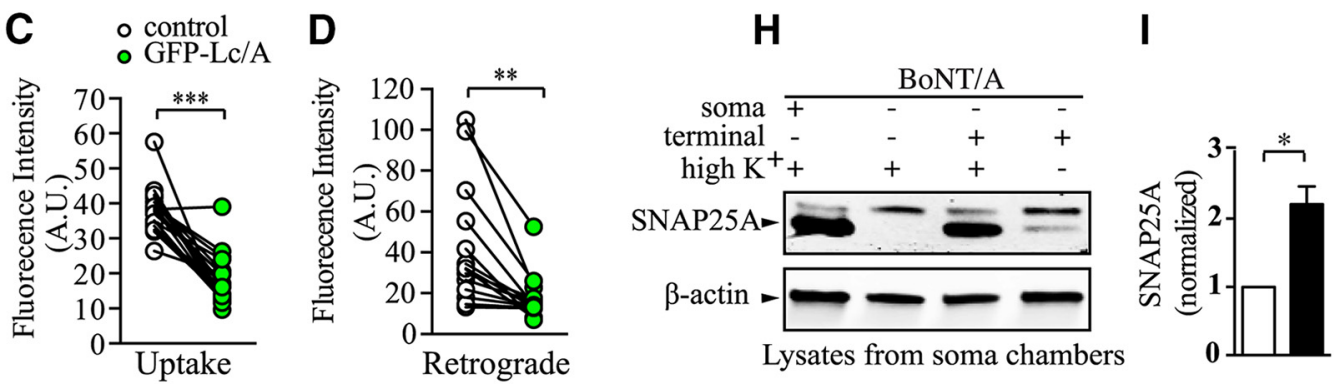

Figure 4. Retrograde BoNT/A holotoxin transport is affected by presynaptic activity. $A-D, B o t h$ BoNT/A-Hc $(H c / A)$ uptake $(A)$ and retrograde trafficking $(B)$ were reduced in GFP-Lc/A-transfected neurons. Hc/A intensity of GFP-LC/A-transfected neurons (box $\boldsymbol{a}$ ) compared with Hc/A intensity of adjacent untransfected neuron (box $\boldsymbol{b}$ ). SNAP25A was used to verify its cleavage in GFP-Lc/Atransfected hippocampal neurons. Scale bar, $20 \mu \mathrm{m}$. C, Quantitative results of $\boldsymbol{A}$. D, Quantitative results of $\boldsymbol{B}$. Data from 3 independent cultures are shown; $n=21$ and 14 for uptake and retrograde, respectively. ${ }^{* *} p<0.01 ;{ }^{* * *} p<0.001$, paired $t$ test. $\boldsymbol{E}$, Hippocampal neurons grown in microfluidic devices were pulsed for 5 min with $100 \mathrm{pm}$ BoNT/A added to the terminal chamber only, after which the long-range effects of BoNT/A were assessed in unstimulated $\left(\right.$ low $\mathrm{K}^{+}$) or stimulated (high $\mathrm{K}^{+}$) conditions by immunofluorescence microscopy of SNAP25A. Chambers without BoNT/A addition were used as the negative control (control). Scale bar, $50 \mu \mathrm{m} . \boldsymbol{F}, \boldsymbol{G}$, Quantitative results of terminal chamber $(\boldsymbol{F})$ and soma chamber $(\boldsymbol{G})$, respectively. Data are from 4 independent cultures; $n=4$. ${ }^{*} p<0.05$, Student's $t$ test. $\boldsymbol{H}$, Representative Western blot of soma chamber lysate samples as described above and soma chamber lysate with BoNT/A added directly to the soma chamber as a positive control (lane 1). $\beta$-actin was used as the loading control. $\boldsymbol{I}$, Quantitative results of $\boldsymbol{H}$, with SNAP25A signal normalized to $\beta$-actin. Data are from 3 independent preparations, $n=3 .{ }^{*} p<$ 0.05 , paired $t$ test.

at different time intervals after high $\mathrm{K}^{+}$stimulation in cultured hippocampal neurons. Because the lipidated form of LC3, LC3-II, has been shown to be a specific marker for autophagic activity in mammals (Komatsu et al., 2005; Tanida et al., 2008), we determined the LC3-II level up to $2 \mathrm{~h}$ after stimulation. We found that LC3-II was significantly increased at $2 \mathrm{~h}$, but not $1 \mathrm{~h}$, after high $\mathrm{K}^{+}$stimulation (Fig. $6 I, J$ ). This result demonstrates that there is a delayed increase in autophagic activity after $\mathrm{K}^{+}$stimulation.

Together, these data indicate that neuronal activity induces the rapid biogenesis of autophagosomes in nerve terminals and that these autophagosomes are subsequently transported to the neuronal soma through fast retrograde axonal trafficking.
Fate of retrogradely transported BoNT/A-Hc in the neuronal soma

Axonal autophagosomes eventually fuse with lysosomes in the cell soma, suggesting that this pool of BoNT/A could be destined for degradation. We therefore examined the colocalization of BoNT/AHc-Atto647 with autophagosome and lysosome markers by immunofluorescence and confocal microscopy at different locations along the BoNT/A-Hc retrograde pathway. Neurons were stimulated for 5 $\mathrm{min}$ in the presence of BoNT/A-Hc-Atto647, followed by a 2-4 h chase in normal medium. They were then fixed and stained with either lysosomal-associated membrane protein 1 (LAMP1) (Wasano and Hirakawa, 1994) or LC3 (Tanida et al., 2008). Consistent with previous reports (Wang et al., 2006), we found that LC3- 


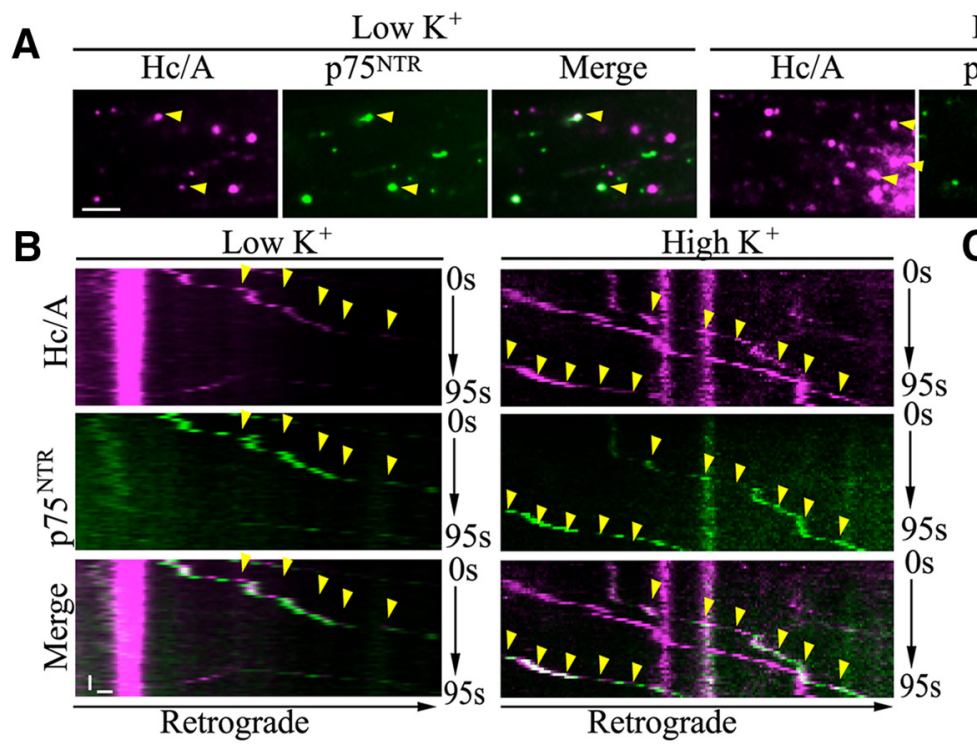

High $\mathrm{K}^{+}$

75 NTR Merge
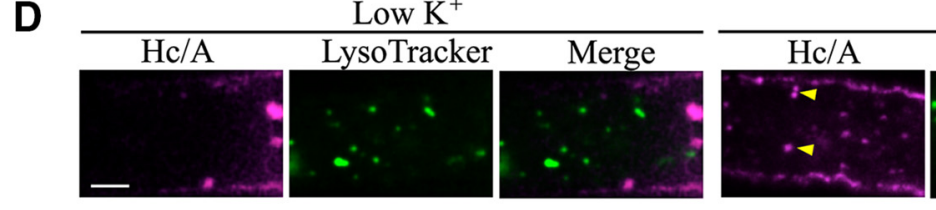

High $\mathrm{K}^{+}$

$\square \mathrm{p} 75^{\mathrm{NTR}}$ negative

- p75 ${ }^{\mathrm{NTR}}$ positive
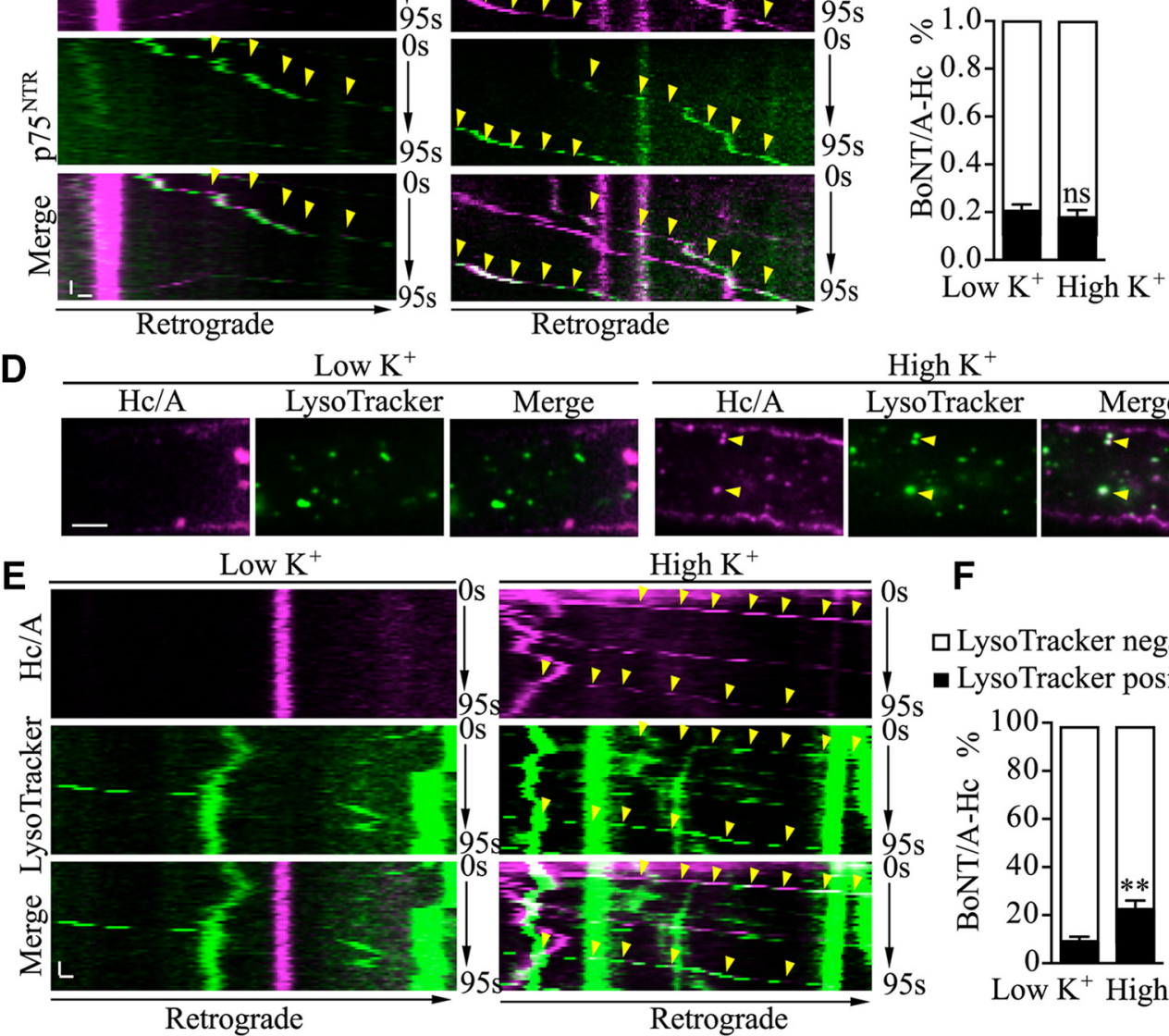

F

$\square$ LysoTracker negative

- LysoTracker positive

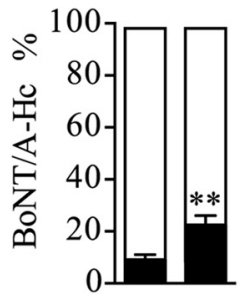

Low $\mathrm{K}^{+}$High $\mathrm{K}^{+}$

Figure 5. BoNT/A-Hc carriers partially colocalize with $\mathrm{p} 75^{\mathrm{NTR}}$ and LysoTracker compartments. A-C, Hippocampal neurons cultured in microfluidic devices were incubated with FITC-p75 NTR antibody for $1 \mathrm{~h}$ at $37^{\circ} \mathrm{C}$ before exposure to stimulated (high $\mathrm{K}^{+}$) or unstimulated (low $\mathrm{K}^{+}$) conditions, with BoNT/A-Hc added to the terminal chamber only. Neurons were chased for $2-4 \mathrm{~h}$ at $37^{\circ} \mathrm{C}$ and the axon channels were imaged. $A$, Images of neurons treated with low $\mathrm{K}^{+}$or high $\mathrm{K}^{+}$. BoNT/A-Hc $(\mathrm{Hc} / \mathrm{A})$ carriers colocalized with p $75^{\mathrm{NTR}}$ are indicated with arrowheads. Scale bar, $5 \mu \mathrm{m} . \boldsymbol{B}$, Kymographs corresponding to the time series described above. Colocalized tracks are indicated with arrowheads. Scale bars: $x$-axis, $1 \mu \mathrm{m} ; y$-axis, 10 s. $\boldsymbol{C}$, Quantification of $\boldsymbol{B}$. Data are from 3 independent preparations; $n=49$ and 46 for low $\mathrm{K}^{+}$and high $\mathrm{K}^{+}$respectively. n.s., Not significant, Student's t test. $\boldsymbol{D}-\boldsymbol{F}$, Hippocampal neurons cultured in microfluidic devices were incubated with LysoTracker Red for $30 \mathrm{~min}$ at $37^{\circ} \mathrm{C}$ before exposure to stimulated (high $\mathrm{K}^{+}$) or unstimulated (low $\mathrm{K}^{+}$) conditions, with BoNT/A-Hc added to the terminal chamber only. Neurons were chased for $2-4 \mathrm{~h}$ at $37^{\circ} \mathrm{C}$ and the axon channels were imaged. $\boldsymbol{D}$, Images of neurons treated with low $\mathrm{K}^{+}$or high $\mathrm{K}^{+}$. BoNT/A-Hc $(\mathrm{Hc} / \mathrm{A})$ carriers colocalized with LysoTracker are indicated with arrowheads. Scale bar, $5 \mu \mathrm{m} . \boldsymbol{E}$, Kymographs corresponding to the time series described above. Colocalized tracks are indicated with arrowheads. Scale bars: $x$-axis, $1 \mu \mathrm{m} ; y$-axis, 10 s. $\boldsymbol{F}$, Quantification of $\boldsymbol{E}$. Data are from 3 independent preparations; $n=51$ and 57 for low $\mathrm{K}^{+}$and high $\mathrm{K}^{+}$, respectively. ${ }^{* *} p<0.01$.

positive puncta, corresponding to autophagosomes, were present in axons located in the neuronal soma (Fig. 7A), nerve terminal chamber (Fig. $7 B$ ), and axon channels (Fig. $7 C$ ). In contrast, LAMP-1positive compartments were highly enriched in the soma (Fig. $7 A$ ), with very few being detected in the axons or nerve terminals (Fig. $7 B$ ), as quantified in Figure $7 D$ and in good agreement with previous studies (Parton et al., 1992).

We next determined the degree of colocalization between BoNT/A-Hc and LAMP1 or LC3 in stimulated and unstimulated neurons. At the level of the soma, $24.9 \pm 2.0 \%$ of the BoNT/AHc-Atto647-positive compartments colocalized with LC3 labeling and $41.9 \pm 4.0 \%$ colocalized with LAMP1 labeling (Fig. 7E), confirming that retrogradely transported autophagosomes fol- low the canonical degradative pathway upon reaching the soma by fusing with acidified lysosomes (Kimura et al., 2007). In the nerve terminal chambers, $35.8 \pm 5.1 \%$ of BoNT/A-Hc labeling colocalized with LC3 labeling whereas only $4.1 \pm 1.0 \%$ colocalized with LAMP1-positive compartments (Fig. 7E), consistent with the incorporation of BoNT/A-Hc-Atto647 into autophagosomes at the nerve terminals.

We then examined the effect of increased presynaptic activity on the formation and transport of autophagosomal BoNT/A-Hcpositive carriers directly in the axon bundles within the channels of microfluidic chambers. In unstimulated conditions, we detected very few LC3- or BoNT/A-Hc-positive carriers and even fewer double-positive compartments $(4.3 \pm 1.4 \%$ double posi- 
A

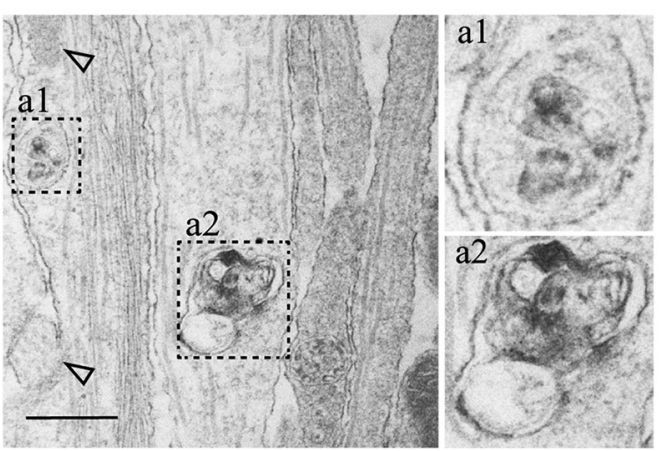

D

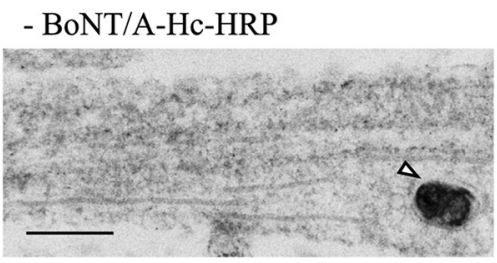

B

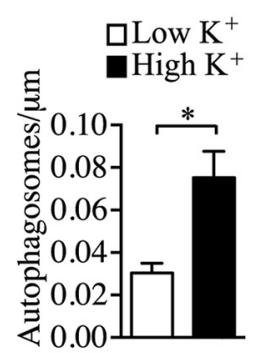

C

+BoNT/A-Hc-HRP

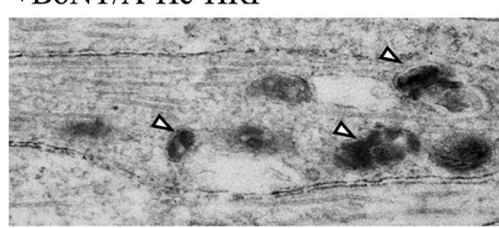

E $\square-$ BoNT/A-Hc-HRP

-BoNT/A-Hc-HRP

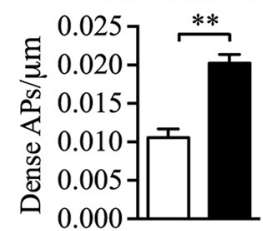

$\mathbf{F}$

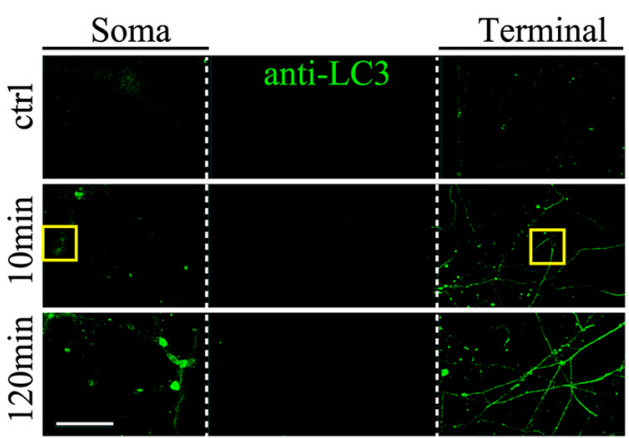

G

H

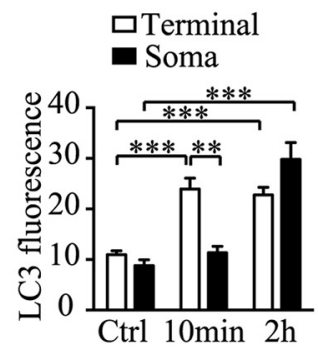

I
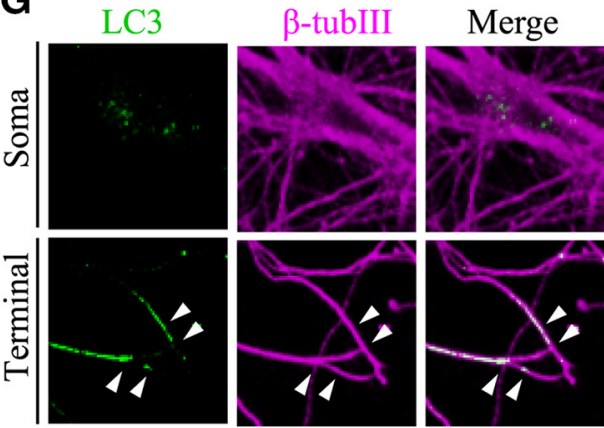

J

Time after high $\mathrm{K}^{+}$pulse (h)
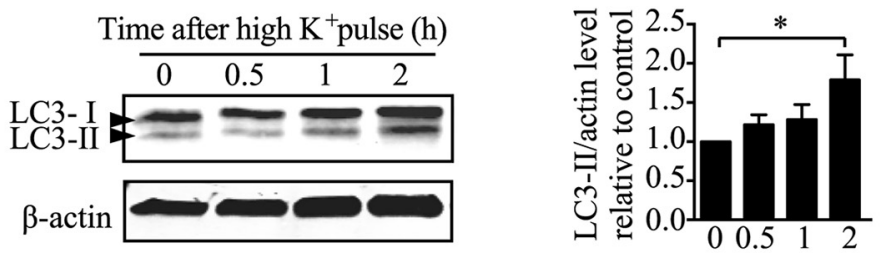

Figure 6. Electron microscopy of axon channels reveals that BoNT/A-Hc is retrogradely transported by autophagosomes. $A$, Representative axon bundles from unlabeled neurons with autophagosomes ( $\boldsymbol{a} 1$ and $\boldsymbol{a} 2$, boxes) and mitochondria (arrowheads) along the microtubule tracks. Scale bar, $500 \mathrm{~nm}$. $\boldsymbol{B}, \boldsymbol{C}$, Quantification of autophagosomes $(\boldsymbol{B})$ and mitochondria $(\boldsymbol{C})$ observed along the axon bundles in the indicated conditions; $n=3$ independent microfluidic devices. ${ }^{*} p<0.05$; n.s. not significant, Student's $t$ test. $\boldsymbol{D}$, Representative electron microscopy of axon bundles of neurons cultured in microfluidic devices with (right) or without (left) BoNT/A-Hc-HRP added to the nerve terminal chambers under high $\mathrm{K}^{+}$conditions. Autophagosomes are indicated by arrowheads. Scale bar, $500 \mathrm{~nm}$. $\boldsymbol{E}$, Quantification of BoNT/A-Hc-HRP-containing autophagosome as shown in $\boldsymbol{D} ; n=3 .{ }^{* *} p<0.01$, Student's $t$ test. $\boldsymbol{F}$, Representative LC3 and $\beta$ III-tubulin immunostaining of hippocampal neurons cultured in microfluidic devices and fixed at indicated times after stimulation. Boxed regions are shown in $\boldsymbol{G}$; arrowheads indicate nerve terminals. $\boldsymbol{H}$, $\mathbf{Q u a n t i f i c a t i o n}$ of the level of LC3 fluorescence normalized to $\beta$ III-tubulin; $n=6$ from 2 independent cultures. ${ }^{* *} p<0.001 ;{ }^{* *} p<0.01$, Student's $t$ test. $I$, Representative LC3 and $\beta$-actin (loading control) immunoblots. $J$, Quantification of $I ; n=3 .{ }^{*} p<0.05$, Student's $t$ test.

tive in the case of LC3 and $14.7 \pm 4.3 \%$ in the case of BoNT/A-Hc; Fig. $7 C$ ). After high $\mathrm{K}^{+}$stimulation, the number of LC3 and BoNT/A-Hc double-positive carriers was significantly increased ( $24.5 \pm 5.8 \%$ of the LC3-positive and $49.7 \pm 6.3 \%$ of the BoNT/ A-Hc-positive compartments contained both labels; Fig. $7 F, G$ ). These data not only suggest that BoNT/A-Hc is incorporated into autophagosomes, but also indicate that autophagosome biogenesis and retrograde transport are upregulated by a single, $5 \mathrm{~min}$ stimulation pulse, consistent with our electron microscopy data (Fig. 6B).

To validate our in vitro pulse-chase data, we performed in vivo experiments in mouse motor neurons. Recombinant mCherry-
BoNT/A-Hc was injected in the right feet of mice and, $24 \mathrm{~h}$ after injection, the level of colocalization of retrogradely transported BoNT/A-Hc with LC3 or LAMP1 was assessed in the cell soma of spinal cord motor neurons (Fig. 8A). Consistent with our in vitro data, we found that the majority $(79.3 \pm 0.02 \%$; Fig. $8 B, D)$ of LC3-positive carriers in the cell bodies of motor neurons also contained BoNT/A-Hc. However, because there were a far greater number of BoNT/A-Hc-positive carriers than LC3positive carriers present in the steady-state motor neuron soma, the number of BoNT/A-Hc-positive carriers that were also positive for LC3 was fairly low ( $5.6 \pm 1.1 \%$ of total; Fig. 8 B, E). A high degree of colocalization was observed between BoNT/A-Hc and 
A

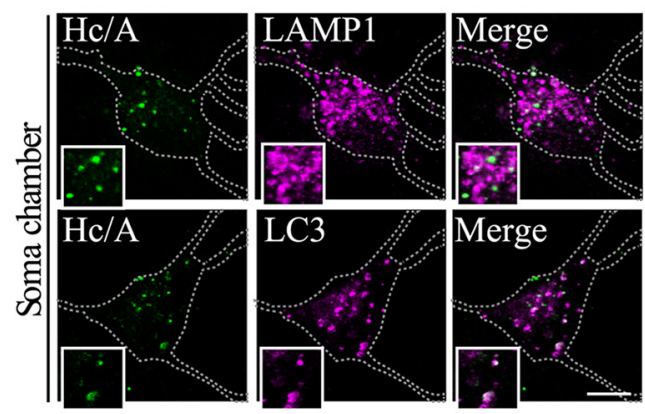

C

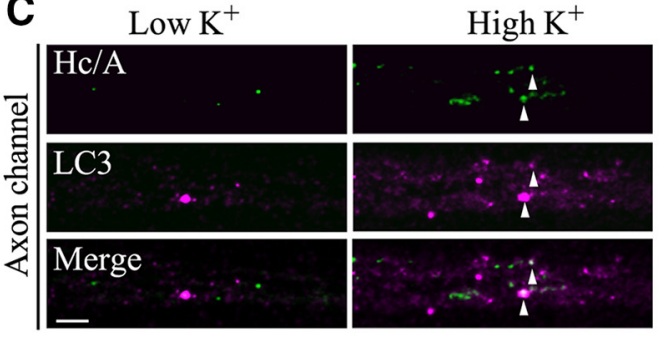

B
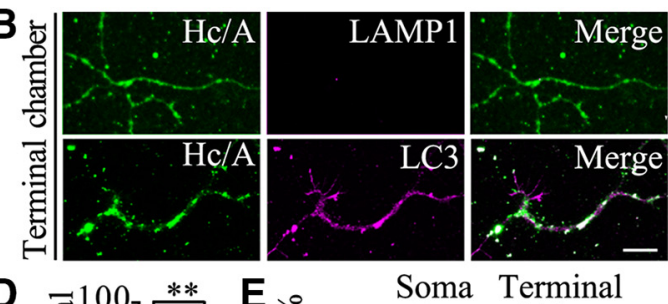

D
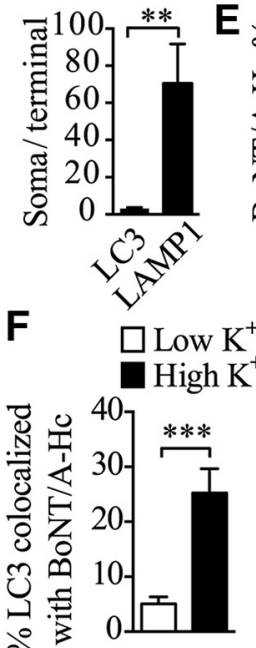

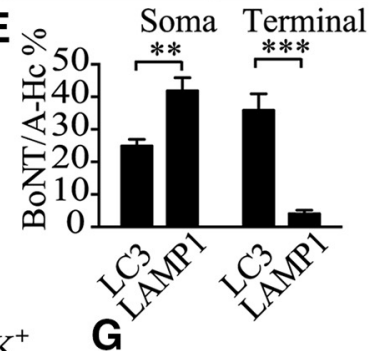

G

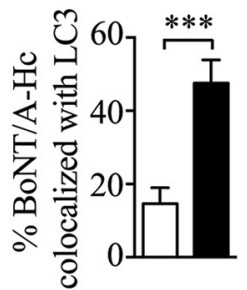

Figure 7. Retrograde BoNT/A-Hc colocalizes extensively with $\mathrm{LC} 3$ along the axon tracks and in the soma. $\boldsymbol{A}-\boldsymbol{D}$, Hippocampal neurons cultured in microfluidic devices were incubated with BoNT/A-Hc-Att0647 (100 nM, Hc/A) for 5 min in high $\mathrm{K}^{+}$, followed by a 2- $4 \mathrm{~h}$ chase in culture medium. Cells were then fixed and processed for immunofluorescence labeling of either LAMP1 or LC3 in the soma $(\boldsymbol{A})$ and terminal $(\boldsymbol{B})$ chambers. Scale bar, $10 \mu \mathrm{m}$. $\boldsymbol{C}$, Neurons were treated as indicated before a $2-4 \mathrm{~h}$ chase, fixation, and labeling of the axon channels. Arrowheads indicate colocalized compartments. Scale bar, $5 \mu \mathrm{m}$. D, Relative distribution of LC3 and LAMP1-label compartments in the soma and nerve terminals. E, Level of colocalization between BoNT/A-Hc-Atto647 (Hc/A) and either LC3 or LAMP1 in soma and terminal chambers. Note the high level of colocalization of BoNT/A-Hc with LAMP1 in the soma, whereas in the terminal chamber, BoNT/A-Hc is mainly associated with LC3 compartments. Data are from 3 independent experiments; $15-49$ fields are analyzed for each group. ${ }^{* *} p<0.01 ; * * 0<0.001$, Student's $t$ test. $\boldsymbol{F}$, $\mathbf{G}$, Level of colocalization (Mander's analysis) between BoNT/A-Hc-Att0647 and LC3 in either low $\mathrm{K}^{+}$-or high $\mathrm{K}^{+}$-treated neurons quantified in the axon channels in the indicated conditions; $n=30$ for low $\mathrm{K}^{+}, n=25$ for high $\mathrm{K}^{+}$. ${ }^{* * *} p<0.001$, Mann-Whitney test.

LAMP1 (50.7 $\pm 2.6 \%$; Fig. $8 C, E)$. Because a far greater number of somatic BoNT/A-Hc-positive carriers were positive for LAMP1 than for LC3, we concluded that autophagosomes play a critical role in transporting BoNT/A-Hc to the soma, where a large proportion of these carriers undergo fusion with lysosomes. These in vivo data further confirmed our in vitro data showing that a significant proportion of presynaptically derived BoNT/A undergoes autophagosome-mediated retrograde trafficking to the neuronal soma. Notably, a nonnegligible proportion of BoNT/A-Hc-positive carriers were negative for both LC3 and LAMP1 in the neuronal soma in both in vitro and in vivo experiments. The nature and pathophysiological relevance of this population is currently unknown (Antonucci et al., 2008; Restani et al., 2012).

BoNT/A-Hc is retrogradely transported in autophagosomes To examine the kinetics and regulation by presynaptic activity of the retrograde autophagosomal transport of BoNT/A-Hc, we performed time-lapse imaging of axons in neurons transfected with GFP-LC3 or RFP-LC3. We first confirmed that GFP-LC3 displayed a cytosolic expression pattern in the soma and a vesicular expression pattern near the nerve terminals (Fig. 9A), as previously reported (Maday and Holzbaur, 2012b). We then analyzed the localization of BoNT/A-Hc after a 5 min uptake pulse in stimulated or unstimulated conditions using GFP-LC3transfected neurons. In unstimulated neurons, only a few retrograde GFP-LC3-positive $(0.52 \pm 0.1 / \mathrm{min}$; Fig. $9 B, D)$ or BoNT/ A-Hc-positive $(1.4 \pm 0.2 / \mathrm{min}$; Fig. $9 B, E)$ carriers were observed and even fewer were colabeled $(0.08 \pm 0.02 / \mathrm{min}$; Fig. $9 B, F)$.
However, after stimulation, the number of GFP-LC3-positive carriers colabeled with BoNT/A-Hc (double-positive carriers) increased $\sim 3.9$-fold $(0.39 \pm 0.09 / \mathrm{min}$; Fig. $9 F)$. The stimulated neurons had a significantly higher percentage of GFP-LC3positive vesicles containing BoNT/A-Hc (Fig. 9G). These data indicate that presynaptic activity increases not only the generation of autophagosomes, but also the sorting of BoNT/A-Hc into this pathway.

Because GFP fluorescence is known to be sensitive to $\mathrm{pH}$, resulting in quenched fluorescence in acidified vesicles (Kimura et al., 2007), we used the pH-resistant RFP-LC3 construct to further investigate the nature of the BoNT/A carriers. We analyzed the number and kinetics of RFP-LC3 carriers in the axons and their level of colocalization with BoNT/A-Hc. As expected, we found that the number of retrograde RFP-LC3-positive carriers was higher than the number of GFP-LC3-positive carriers, confirming that a significant proportion of the retrograde, autophagosome-derived carriers were acidified (Fig. 9C,D), as previously reported (Lee et al., 2011; Maday and Holzbaur, 2012b). Consistent with our data using GFP-LC3, we found the same activity-dependent increase in the number of RFP-LC3positive retrograde carriers (from $0.4 \pm 0.08 / \mathrm{min}$ to $1.5 \pm 0.3 /$ min; Fig. 9D), BoNT/A-Hc-positive carriers (Fig. 9E), and RFPLC3 and BoNT/A-Hc double-positive carriers ( $\sim 3.5$-fold) (Fig. $9 F$ ) and also an increase in the percentage of double-positive carriers relative to the total number of RFP-LC3-positive vesicles (Fig. 9G).

One of the first stages in the maturation of autophagosomes is their fusion with acidified but nondegradative components of the 
A

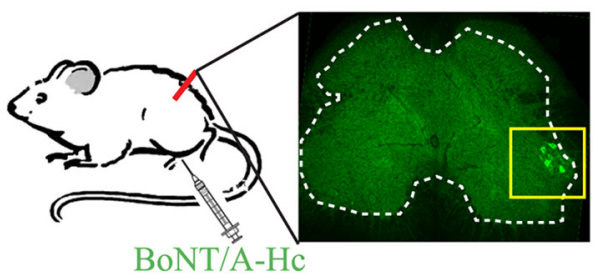

B
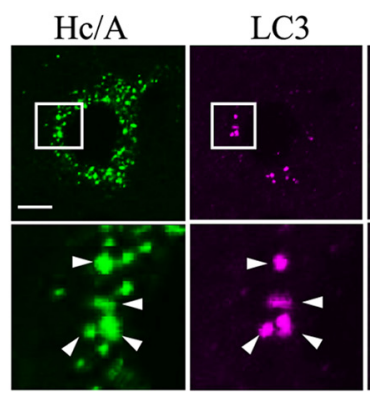

Merge

C

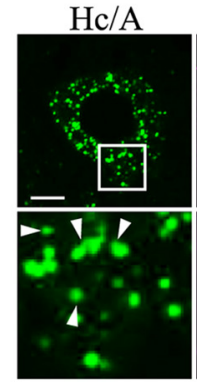

LAMP1
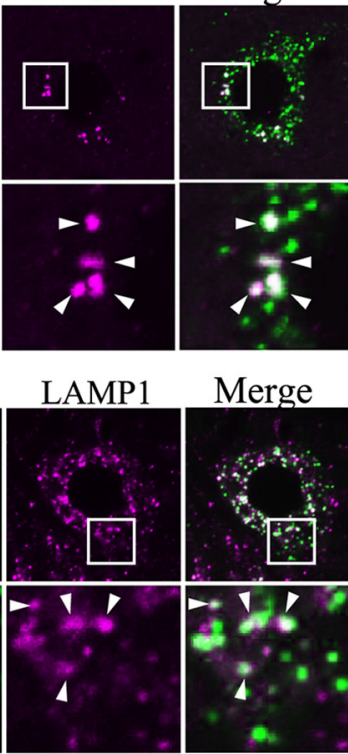

D

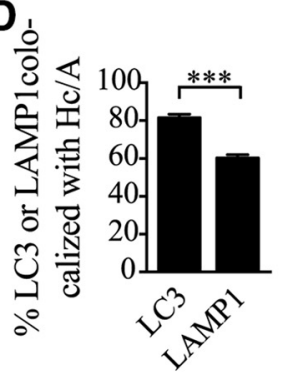

E

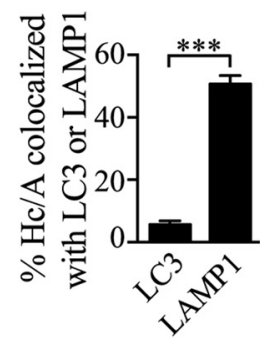

Figure 8. Retrogradely transported mCherry-BoNT/A-Hc accumulates in lysosomes from spinal cord motor neuron soma after paw injection. $A$. Schematic of paw injection of mCherryBoNT/A-Hc $(\mathrm{Hc} / \mathrm{A})$ in vivo. Twenty-four hours after injection, mice were killed and perfused. The spinal cord was then dissected and sliced for immunofluorescence processing. The level of colocalization of mCherry-BoNT/A-Hc wi'th LC3 or LAMP1 was assessed in the cell soma of ipsilateral spinal cord motor neurons. $\boldsymbol{B}, \boldsymbol{C}$, Representative images of mCherry-BoNT/A-Hc colocalized with LC3 $(\boldsymbol{B})$ and LAMP1 $(\boldsymbol{C})$ in the soma of spinal cord motor neurons. Scale bar, 10 $\mu m . \boldsymbol{D}, \boldsymbol{E}$, Mander's ratio of colocalization between BoNT/A-Hc and LAMP1 or LC3, respectively. Data are from 3 independent experiments; $n=52$ for LC 3 staining, $n=59$ for LAMP1 staining. *** $p<0.001$, Student's $t$ test.

endosomal system to generate intermediate compartments termed amphisomes that subsequently fuse with lysosomes in the cell soma. To elucidate whether neuronal activity affects this process, we used tandem fluorescently tagged LC3 (tFP-LC3: mRFPEGFP-LC3). In axons, the maturation of autophagosomes is reflected by changes in the ratio of RFP/GFP fluorescence, in that LC3-positive vesicles retaining both GFP and RFP fluorescence are autophagosomes (Fig. 10A,A1,A3, boxes), whereas LC3positive vesicles displaying only RFP fluorescence are amphisomes and/or autolysosomes (Fig. 10A,A2,A4, boxes) (Maday and Holzbaur, 2012b; Martin et al., 2013). We found that, in the cell body of neurons, stimulation increased the number of autolysosomes, which are the most prevalent LC3-positive compartment, but had no obvious effect on the number of autophagosomes (Fig. 10B). In contrast, autophagosomes were more abundant in the nerve terminals (Fig. $10 A, A 1, A 3$, boxes, $B)$. When we subsequently analyzed the distribution of BoNT/ A-Hc between autolysosomes and autophagosomes in stimulated and unstimulated neurons, no significant difference was observed (Fig. 10C), suggesting that presynaptic activity promotes autophagosome maturation without affecting the sorting of BoNT/A-Hc within this pathway. In agreement with previous reports showing that LC3 retrograde trafficking can be increased by excitotoxic stimuli (Katsumata et al., 2010), our results clearly demonstrate that the number of presynaptically generated autophagosomes is upregulated by depolarizing stimulation and that these autophagosomes undergo efficient maturation in the cell soma. This upregulated retrograde flux of autophagosomes is a major trafficking pathway mediating the activity-dependent retrograde transport of BoNT/A-Hc.

Biogenesis and maturation of autophagosomes is required for BoNT/A-Hc retrograde trafficking

Finally, to test whether autophagosomes play a role in BoNT/ A-Hc retrograde trafficking, we examined the effects of two different pharmacological autophagy inhibitors on this process. Bafilomycin $A_{1}$, a potent and specific inhibitor of vacuolar $\mathrm{H}^{+}$ ATPase, inhibits the acidification of autophagosomes and blocks the later stage of autophagy (Yamamoto et al., 1998). In contrast, wortmannin, a pan PI3-kinase inhibitor (Meunier et al., 2005; Wen et al., 2008; Wen et al., 2011), inhibits autophagosome formation and blocks the early stage of autophagy (Blommaart et al., 1997). Hippocampal neurons cultured in microfluidic devices were incubated with wortmannin $(40 \mathrm{nM})$, bafilomycin $\mathrm{A}_{1}(50$ $\mathrm{nM}$ ), or DMSO (vehicle) for $0.5 \mathrm{~h}$ before a pulse of BoNT/A-Hc labeling at the nerve terminals under high $\mathrm{K}^{+}$stimulation. After a 2-4 h chase in the continued presence of wortmannin or bafilomycin $A_{1}$, we found that both autophagy inhibitors significantly reduced the frequency of BoNT/A-Hc retrograde carriers detected along the axon channels, as indicated by a significant decrease in retrograde BoNT/A-Hc carrier numbers in treated axons compared with the group incubated with DMSO (Fig. 11A,B).

These data demonstrate that, in hippocampal neurons, the autophagy pathway is required for the activity-dependent retrograde axonal trafficking of BoNT/A-Hc. This result is also consistent with our data showing that increasing presynaptic activity upregulates both axonal autophagy flux and BoNT/A-Hc retrograde trafficking (Figs. 6,9) and supports the notion that a substantial portion of activity-dependent BoNT/A-Hc retrograde transport is mediated by nerve-terminal-initiated autophagosome flux.

\section{Discussion}

In the present study, we demonstrate that presynaptic activity in hippocampal neurons increases the retrograde transport of BoNT/ A-Hc, as well as active BoNT/A holotoxin, from the nerve terminal to the cell soma. Detailed characterization of the activity-dependent retrograde trafficking of BoNT/A-Hc has shown that a significant proportion of this traffic is mediated by autophagosomal flux both in vitro and in vivo. We reveal that raising presynaptic activity upregulates the biogenesis of autophagosomes in a process initiated at the nerve terminal and results in increased autophagosomal delivery of the toxin cargo to the soma. 
A

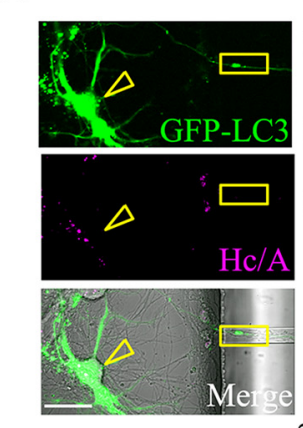

C

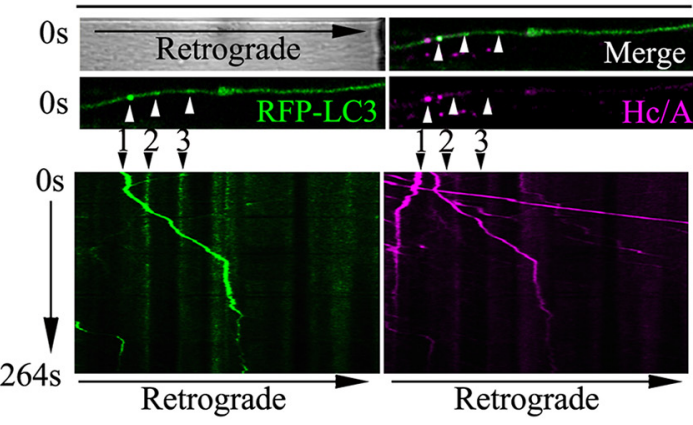

Low $\mathrm{K}^{+}$

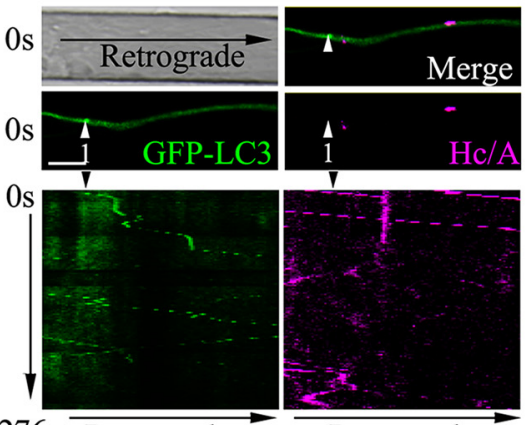

$276 \mathrm{~s}$ Retrograde

Retrograde
$\operatorname{High~} \mathrm{K}^{+}$

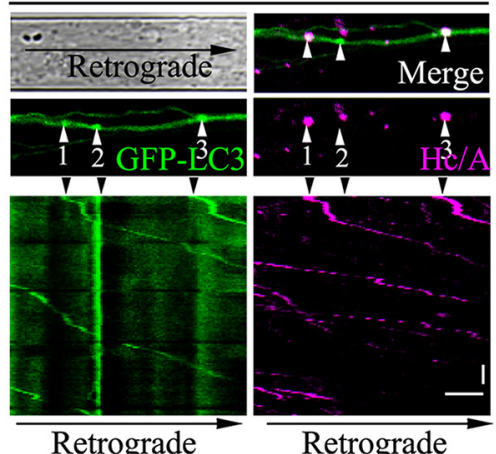

Retrograde

$\operatorname{High~K}^{+}$
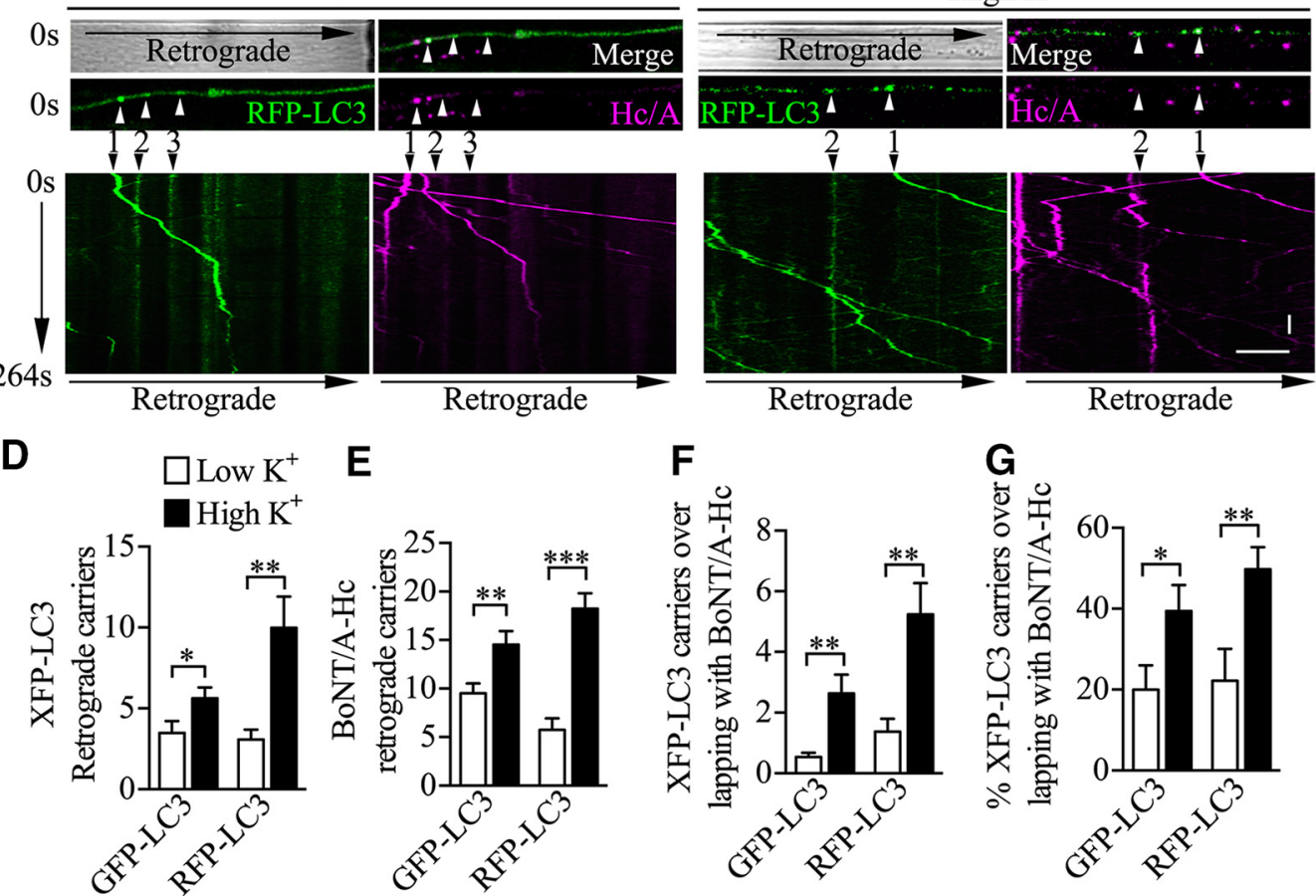

Figure 9. Presynaptic activity increases LC3-mediated BoNT/A-Hc axonal retrograde trafficking. GFP-LC3- or RFP-LC3-transfected neurons cultured in microfluidic devices were incubated with BoNT/A-Hc-Atto647 (100 nm) for 5 min in either low- or high $\mathrm{K}^{+}$medium, followed by a 2-4 $\mathrm{h}$ chase in culture. $A$, Representative images of axons extending into the nerve terminal chambers labeled with both BoNT/A-Hc-Atto647 (Hc/A, middle) and GFP-LC3 (top); arrowhead points to a soma. Time-lapse imaging was performed in regions proximal to the soma chamber (yellow boxes). Scale bar, $30 \mu \mathrm{m}$. B, Top, Time-lapse imaging of BoNT/A-Hc-Att0647 (Hc/A) and GFP-LC3 in axons. Bottom, Kymographs of the same axons showing carrier movement over $276 \mathrm{~s}$. Arrowheads point to moving carriers. C, Top, Time-lapse imaging of BoNT/A-Hc-Atto647 (HC/A) and RFP-LC3 in axons. Bottom, Kymographs of the same axons showing carrier movement over $264 \mathrm{~s}$. Arrowheads point to moving carriers. Scale bars: $x$-axis, $10 \mu \mathrm{m}$; $y$-axis, 10 s. D-G, Quantification of the number of XFP-LC3 (GFP-LC3 or RFP-LC3, respectively, as labeled on the $x$-axis; $\boldsymbol{D}$ ), BoNT/A-Hc-Atto647 (E), and BoNT/A-Hc/XFP-LC3 double-positive vesicles $(\boldsymbol{F})$ detected over $400 \mathrm{~s}$ in the indicated conditions. G, Percentage of XFP-LC3 vesicles containing BoNT/A-Hc. GFP-LC3-transfected groups: $n=43$ for low $\mathrm{K}^{+}, n=51$ for high $\mathrm{K}^{+}$. RFP-LC3-transfected groups: $n=27$ for low $\mathrm{K}^{+}, n=30$ for high $\mathrm{K}^{+} .{ }^{*} p<0.05{ }^{* *} p<0.01 ;{ }^{* * *} p<0.001$, Mann-Whitney test.

\section{Retrograde transport of BoNT/A-Hc}

Unlike tetanus neurotoxin, which depends on retrograde axonal transport to mediate its potent blockade of inhibitory neurotransmission (von Bartheld, 2004; Deinhardt and Schiavo, 2005), the classically accepted cellular target of the BoNTs is the peripheral motor nerve terminal, where they induce flaccid paralysis by blocking acetylcholine release (Meunier et al., 2002). However, retrograde transport of some BoNT types has recently been discovered in vivo and in cultured neurons, revealing that these neurotoxins also undergo long-distance retrograde trafficking and transcytosis in central neurons (Antonucci et al., 2008; Restani et al., 2011; Restani et al., 2012), which is consistent with the central effects of BoNT/A observed in some patients (Caleo et al., 2009). The fact that BoNT/A uptake into the nerve terminals is facilitated by stimulation (Dong et al., 2006; Harper et al., 2011) and that the toxin is retrogradely transported provided a unique opportunity to address whether BoNT/A retrograde flux is also controlled by presynaptic activity.

After BoNTs enter nerve terminals via receptor-mediated endocytosis, the exposure of the BoNT holotoxin to the acidic $\mathrm{pH}$ of the synaptic vesicles induces release of the Lc protease into the cytosol while the binding domain of the Hc remains on the synaptic vesicle lipid bilayer (Koriazova and Montal, 2003; Pirazzini et al., 2014). The Hc-binding domain can be therefore used as a reliable tool to monitor the binding, uptake, and retrograde trafficking of the parental BoNT/A in motor and hippocampal neurons (Harper et al., 2011; Restani et al., 2012). Here, we used BoNT/A-Hc and demonstrated that, in central neurons cultured in microfluidic chambers, the frequency of BoNT/A-Hc-positive 
A
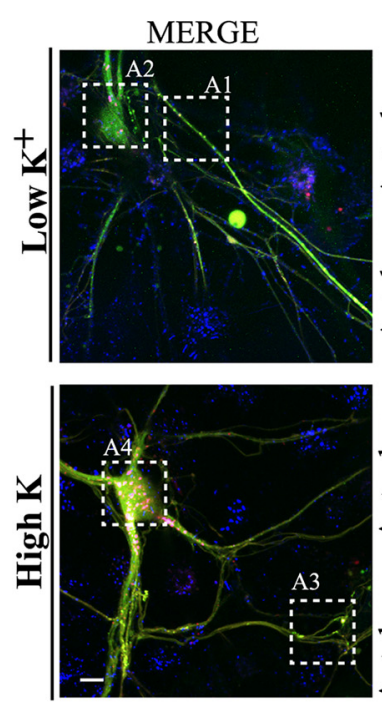
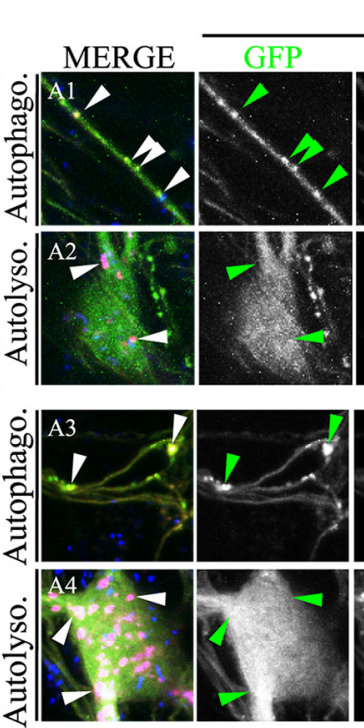

tFP-LC3
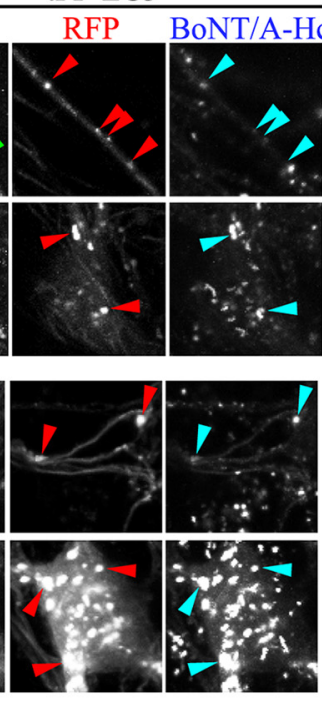

$\begin{array}{ll}\text { B } & \square \text { Low K } \\ & \text { High K }\end{array}$

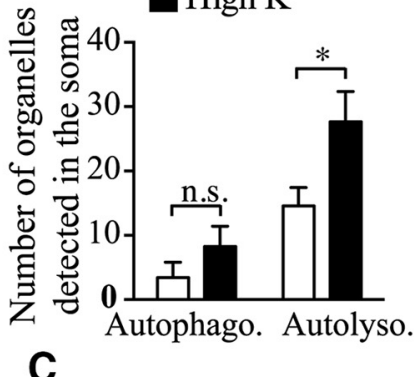

$C$

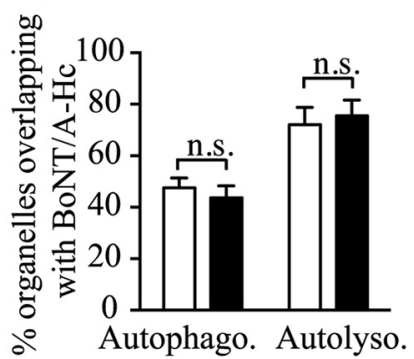

Figure 10. Presynaptic activity does not influence the fate of retrogradely transported BoNT/A-Hc upon reaching the soma. Tandem fluorescently tagged LC3 (tFP-LC3: mRFP-EGFP-LC3)transfected neurons were incubated with BoNT/A-Hc-Att0647 (100 nm) for 5 min in either low or high $\mathrm{K}^{+}$medium, followed by a $2-4 \mathrm{~h}$ chase in culture medium. $\boldsymbol{A}$, Representative images of autophagosome (Autophag. $\boldsymbol{A} \mathbf{1}$ and $\boldsymbol{A} \mathbf{3}$ boxes) and autolysosome (Autolyso., $\boldsymbol{A} \mathbf{2}$ and $\boldsymbol{A 4}$ boxes) and in the proximal axons $(\boldsymbol{A 1}, \boldsymbol{A} \mathbf{3})$ and soma $(\boldsymbol{A 2}, \boldsymbol{A 4})$ in the indicated conditions. Arrowheads indicate tFP-LC3-positive vesicles. Scale bar, $10 \mu \mathrm{m}$. $\boldsymbol{B}$, Quantification of the number of somatic autolysosomes and autophagosomes in the indicated conditions. $\boldsymbol{C}$, Percentage of somatic autophagosomes and autolysosomes containing BoNT/A-Hc in the indicated conditions. Data are from 3 independent cultures; $n=9$ for low $\mathrm{K}^{+} ; n=11$ for high $\mathrm{K}^{+}$. ${ }^{*} p<0.05$, Mann-Whitney test.

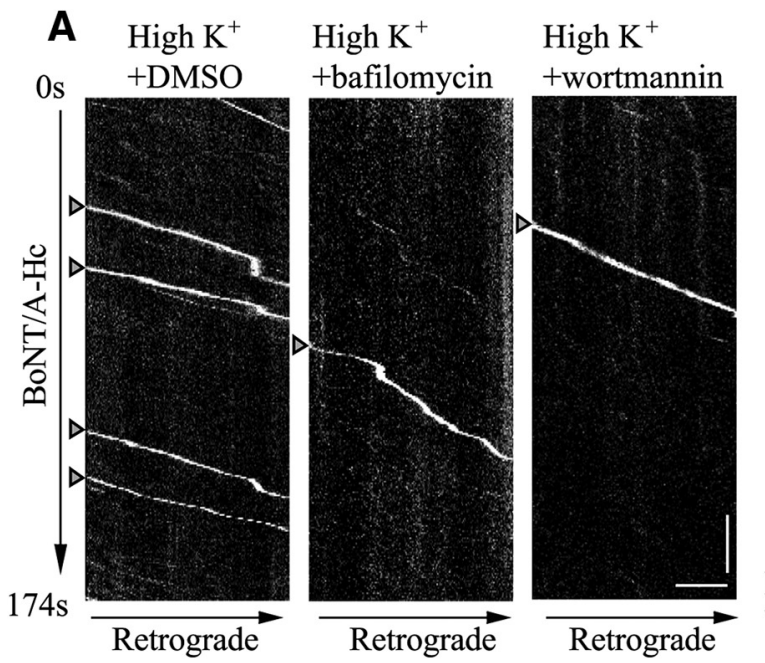

B

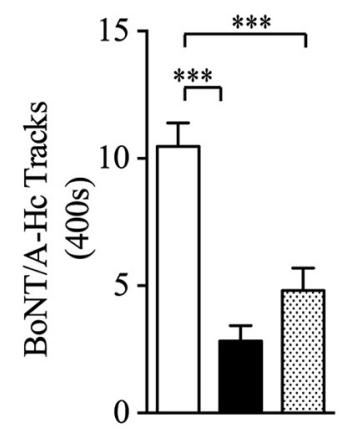

High $\mathrm{K}^{+}+\quad+\quad+$ Bafilomycin - $\quad+\quad-$ Wortmannin - $\quad+$

Figure 11. Activity-dependent BoNT/A-Hc retrograde flux is blocked by autophagy inhibitors. Hippocampal neurons cultured in microfluidic devices were incubated with wortmannin ( $40 \mathrm{~nm}$ ), bafilomycin $A_{1}(50 \mathrm{~nm})$, or DMSO for $0.5 \mathrm{~h}$ before pulse-chase BoNT/A-Hc labeling at nerve terminals under high $\mathrm{K}^{+}$stimulation. $A$, Kymographs of BoNT/A-Hc carriers in DMSO vehicle- (left), bafilomycin $A_{1}$ - (middle), or wortmannin (right)-treated axon channels over $174 \mathrm{~s}$. Retrograde tracks are marked with arrowheads. Scale bars: $x$-axis, $10 \mu \mathrm{m} ; y$-axis, 20 s. $B$, Quantification of BoNT/A-Hc retrograde carrier numbers in corresponding neurons, as shown in $A ; n=53$ DMSO; $n=20$ bafilomycin $A_{1} ; n=56$ wortmannin, ${ }^{* * *} p<0.001$, Mann-Whitney test.

retrograde carriers is greatly increased upon stimulation, whereas the average speed $(0.8 \mu \mathrm{m} / \mathrm{s})$ and behavior of these carriers is unaffected. Because the BoNT/A-Hc probe was used at a high concentration here $(100 \mathrm{nM})$, we also examined the fate of BoNT/ A-Hc at a more pathologically relevant concentration (1 nM) using single-particle tracking. We found that, at this concentration, BoNT/A-Hc was also recruited to fast axonal retrograde trafficking. The values found for the average speed are in good agreement with a previous report (Restani et al., 2012) regardless of the concentration of BoNT/A-Hc used. We also used the same protocol to determine whether the retrograde trafficking of BoNT/A holotoxin is regulated by presynaptic activity, revealing that BoNT/A holotoxin internalized from the nerve terminal reached the soma and remained catalytically active, as reflected by cleavage of SNAP25 in the soma chamber. Importantly, we further found that the amount of cleaved SNAP25 in the soma chamber increased in response to a stimulatory pulse of high $\mathrm{K}^{+}$, indicating that this was sufficient to increase the amount of active BoNT/A transported to the soma that eventually reaches the afferent synapses after transcytosis. We can only assume that this was the result of BoNT/A activity after transcytosis in the afferent terminals because of the extent of SNAP25 cleavage found in the soma chamber from our Western blot analysis. However, more work is warranted to investigate whether some cleaved SNAP25 has also been transported to the soma for degradation and/or if some active Lc accessed the soma cytosol after axonal transport. More work is needed to assess whether and how transcytosis occur after retrograde transport, a pathway that has remained enigmatic. Although we found that approximately half of the BoNT/A-Hc is transported via autophagosomes, we cannot exclude the possibility that the active holotoxin might be transported via a parallel pathway that is also upregulated by presynaptic activity. Further studies using the holotoxin are also 
warranted to determine whether inhibiting autophagy can prevent the active toxin from acting in the soma chamber.

\section{Retrograde pathways that mediate transport of BoNT/A-Hc in neurons}

Axonal retrograde transport of target-derived materials to the distal soma is crucial for the survival and function of neurons, the most classical example of which is the retrograde transport of neurotrophin factors and their receptors (i.e., nerve growth factor and p75 ${ }^{\mathrm{NTR}}$ ) via signaling endosomes (Zweifel et al., 2005). Recent studies in mammalian neurons have revealed a subpopulation of virulence factors (i.e., rabies virus, tenanus toxin, and BoNTs) that hijack this long-range retrograde pathway to reach the soma, but whether these carriers are acidified remains controversial. In motor neurons, tetanus toxin and BoNTs hijack nonacidified $\mathrm{p} 75^{\mathrm{NTR}}$-transporting organelles (Lalli and Schiavo, 2002; Restani et al., 2012), but in dorsal root ganglion neurons, the $\mathrm{p} 75^{\mathrm{NTR}}$-binding rabies virus is delivered to the soma via acidified p $75^{\text {NTR }}$ carriers (Gluska et al., 2014). In our study using BoNT/A-Hc as a probe for synapse-derived vesicles, we identified a portion of the retrograde carriers that colocalized with $\mathrm{p} 75^{\mathrm{NTR}}$ and were not affected by presynaptic activity (Fig. $5 A-C$ ). However, some of BoNT/A-Hc localized to acidified organelles. Interestingly, this population was significantly increased with high $\mathrm{K}^{+}$ stimulation (Fig. $5 D-F$ ), suggesting the existence of presynapticactivity-dependent sorting mechanisms that recruit BoNT/A-Hc to acidified compartments.

In axons, autophagosomes are one of the best-defined acidified compartments, mediating the degradation of terminal-derived cargoes after long-distance axonal retrograde transport (Lee et al., 2011; Maday and Holzbaur, 2012b). Although the content of autophagosomes can be derived from many cellular sources (Weidberg et al., 2011), the main source of the autophagosomes within the nerve terminals of neurons is the endocytic pathway (Fader and Colombo, 2009; Yang et al., 2013), which converges with the autophagic pathway through late endosome/multivesicular bodies or early endosome fusion to form the singlemembrane amphisome (Berg et al., 1998; Fader and Colombo, 2009). In this study, we found that the proportion of BoNT/A-Hc carriers overlapping with either autophagosomes or acidified compartments was significantly increased after synaptic activation, suggesting that there might be sorting machinery between the autophagic pathway and its upstream endocytic pathway, a process that is highly regulated by neuronal activity. However, it is still unknown how this machinery bifurcates to distribute endocytosed cargoes between nonacidified signaling endosomes and acidified autophagosomes. It could be worthwhile to analyze the sorting mechanism for terminal-endocytosed cargoes destined for different retrograde pathways given the importance of retrograde transport, which is essential for neuronal survival and function (Maday and Holzbaur, 2012b; Nixon, 2013; Yang et al., 2013).

\section{Regulation of presynaptic autophagy of BoNT/A-Hc by presynaptic activity}

Autophagy in the mammalian brain is a highly inducible pathway that is normally kept at a low basal level, but can be rapidly activated in response to environmental stimuli (Mizushima et al., 2001; Koushika et al., 2004; Xie and Klionsky, 2007). In hippocampal pyramidal neurons, depolarization induces the activation of autophagy, which promotes presynaptic AMPA receptor degradation (Shehata et al., 2012). In addition to postsynaptic autophagy, an excitotoxic insult has been found to increase the retrograde autophagosome flux in cerebellar explant axons (Katsumata et al., 2010). With real-time imaging in live neurons, we revealed an increase in the retrograde flux of endogenous and fluorescently tagged LC3-positive compartments and, using electron microscopy, we were able to identify an increased number of double-membrane autophagosome compartments in axons 2-4 $\mathrm{h}$ after stimulation. Because a major component of these autophagosomes is synaptically derived BoNT/A-Hc, our data indicate that presynaptic activity upregulates the incorporation of presynaptically derived endocytic cargoes into retrogradely targeted autophagosomes. This suggests that autophagy may play a physiological role in restoring neuronal homeostasis, which is transiently disturbed by neuronal activity.

In neurons, the pharmacological inhibitors bafilomycin $A_{1}$ and wortmannin can be used to block different steps of the autophagy pathway, which is the key retrograde transport pathway for the axon degradation of derived material (Wang et al., 2006). Consistent with this, we found that inhibition of autophagy using either inhibitor also blocked BoNT/A-Hc retrograde flux, suggesting that autophagic activation is required for BoNT/A-Hc retrograde trafficking and further supporting the idea that BoNT/ A-Hc undergoes retrograde transport through autophagosomes. More experiments will be needed to determine whether such pharmalogical interventions could also prevent the retrograde transport and transcytosis of the holotoxin in the soma chamber in vitro and in vivo.

In conclusion, we have demonstrated that BoNT/A-Hc undergoes retrograde trafficking after endocytic uptake at the synapse. Importantly, this retrograde flux is significantly upregulated by presynaptic activity. A substantial proportion of retrograde BoNT/A-Hc-positive carriers are colabeled by the autophagosome marker LC3 both in vitro and in vivo. Similarly, LC3-positive carrier flux also shows an activity-dependent increase, suggesting that autophagy plays a key regulatory role in restoring presynaptic homeostasis. Because neuronal autophagy defects underlie many neurodegenerative conditions, understanding the precise physiological role that this process plays in the brain is of considerable importance.

\section{References}

Antonucci F, Rossi C, Gianfranceschi L, Rossetto O, Caleo M (2008) Longdistance retrograde effects of botulinum neurotoxin A. J Neurosci 28: 3689-3696. CrossRef Medline

Bajohrs M, Rickman C, Binz T, Davletov B (2004) A molecular basis underlying differences in the toxicity of botulinum serotypes A and E. EMBO Rep 5:1090-1095. CrossRef Medline

Benoit RM, Frey D, Hilbert M, Kevenaar JT, Wieser MM, Stirnimann CU, McMillan D, Ceska T, Lebon F, Jaussi R, Steinmetz MO, Schertler GF, Hoogenraad CC, Capitani G, Kammerer RA (2014) Structural basis for recognition of synaptic vesicle protein $2 \mathrm{C}$ by botulinum neurotoxin $\mathrm{A}$. Nature 505:108-111. Medline

Berg TO, Fengsrud M, Strømhaug PE, Berg T, Seglen PO (1998) Isolation and characterization of rat liver amphisomes: evidence for fusion of autophagosomes with both early and late endosomes. J Biol Chem 273: 21883-21892. CrossRef Medline

Binz T, Kurazono H, Wille M, Frevert J, Wernars K, Niemann H (1990) The complete sequence of botulinum neurotoxin type $\mathrm{A}$ and comparison with other clostridial neurotoxins. J Biol Chem 265:9153-9158. Medline

Blasi J, Chapman ER, Link E, Binz T, Yamasaki S, De Camilli P, Südhof TC, Niemann H, Jahn R (1993) Botulinum neurotoxin A selectively cleaves the synaptic protein SNAP-25. Nature 365:160-163. CrossRef Medline

Blommaart EF, Krause U, Schellens JP, Vreeling-Sindelárová H, Meijer AJ (1997) The phosphatidylinositol 3-kinase inhibitors wortmannin and LY294002 inhibit autophagy in isolated rat hepatocytes. Eur J Biochem 243:240-246. CrossRef Medline

Bronfman FC, Tcherpakov M, Jovin TM, Fainzilber M (2003) Ligand- 
induced internalization of the p75 neurotrophin receptor: a slow route to the signaling endosome. J Neurosci 23:3209-3220. Medline

Caleo M, Antonucci F, Restani L, Mazzocchio R (2009) A reappraisal of the central effects of botulinum neurotoxin type A: by what mechanism? J Neurochem 109:15-24. CrossRef Medline

Colasante C, Rossetto O, Morbiato L, Pirazzini M, Molgó J, Montecucco C (2013) Botulinum neurotoxin type A is internalized and translocated from small synaptic vesicles at the neuromuscular junction. Mol Neurobiol 48:120-127. CrossRef Medline

Couesnon A, Shimizu T, Popoff MR (2009) Differential entry of botulinum neurotoxin A into neuronal and intestinal cells. Cell Microbiol 11:289308. CrossRef Medline

Deinhardt K, Schiavo G (2005) Endocytosis and retrograde axonal traffic in motor neurons. Biochem Soc Symp 72:139-150. Medline

Dolly JO, Black J, Williams RS, Melling J (1984) Acceptors for botulinum neurotoxin reside on motor nerve terminals and mediate its internalization. Nature 307:457-460. CrossRef Medline

Dong M, Yeh F, Tepp WH, Dean C, Johnson EA, Janz R, Chapman ER (2006) SV2 is the protein receptor for botulinum neurotoxin A. Science 312:592596. CrossRef Medline

Ekong TA, Feavers IM, Sesardic D (1997) Recombinant SNAP-25 is an effective substrate for Clostridium botulinum type A toxin endopeptidase activity in vitro. Microbiology 143:3337-3347. CrossRef Medline

Fader CM, Colombo MI (2009) Autophagy and multivesicular bodies: two closely related partners. Cell Death Differ 16:70-78. CrossRef Medline

Foran PG, Davletov B, Meunier FA (2003) Getting muscles moving again after botulinum toxin: novel therapeutic challenges. Trends Mol Med 9:291-299. CrossRef Medline

Fu Z, Chen C, Barbieri JT, Kim JJ, Baldwin MR (2009) Glycosylated SV2 and gangliosides as dual receptors for botulinum neurotoxin serotype F. Biochemistry 48:5631-5641. CrossRef Medline

Gluska S, Zahavi EE, Chein M, Gradus T, Bauer A, Finke S, Perlson E (2014) Rabies virus hijacks and accelerates the p75NTR retrograde axonal transport machinery. PLoS Pathog 10:e1004348. CrossRef Medline

Harper CB, Martin S, Nguyen TH, Daniels SJ, Lavidis NA, Popoff MR, Hadzic G, Mariana A, Chau N, McCluskey A, Robinson PJ, Meunier FA (2011) Dynamin inhibition blocks botulinum neurotoxin type A endocytosis in neurons and delays botulism. J Biol Chem 286:35966-35976. CrossRef Medline

Kabeya Y, Mizushima N, Ueno T, Yamamoto A, Kirisako T, Noda T, Kominami E, Ohsumi Y, Yoshimori T (2000) LC3, a mammalian homologue of yeast Apg8p, is localized in autophagosome membranes after processing. EMBO J 19:5720-5728. CrossRef Medline

Katsumata K, Nishiyama J, Inoue T, Mizushima N, Takeda J, Yuzaki M (2010) Dynein- and activity-dependent retrograde transport of autophagosomes in neuronal axons. Autophagy 6:378-385. CrossRef Medline

Kechkar A, Nair D, Heilemann M, Choquet D, Sibarita JB (2013) Real-time analysis and visualization for single-molecule based super-resolution microscopy. PLoS One 8:e62918. CrossRef Medline

Kimura S, Noda T, Yoshimori T (2007) Dissection of the autophagosome maturation process by a novel reporter protein, tandem fluorescenttagged LC3. Autophagy 3:452-460. CrossRef Medline

Komatsu M, Waguri S, Ueno T, Iwata J, Murata S, Tanida I, Ezaki J, Mizushima N, Ohsumi Y, Uchiyama Y, Kominami E, Tanaka K, Chiba T (2005) Impairment of starvation-induced and constitutive autophagy in Atg7-deficient mice. J Cell Biol 169:425-434. CrossRef Medline

Koriazova LK, Montal M (2003) Translocation of botulinum neurotoxin light chain protease through the heavy chain channel. Nat Struct Biol 10:13-18. CrossRef Medline

Koushika SP, Schaefer AM, Vincent R, Willis JH, Bowerman B, Nonet ML (2004) Mutations in Caenorhabditis elegans cytoplasmic dynein components reveal specificity of neuronal retrograde cargo. J Neurosci 24:39073916. CrossRef Medline

Lalli G, Schiavo G (2002) Analysis of retrograde transport in motor neurons reveals common endocytic carriers for tetanus toxin and neurotrophin receptor p75NTR. J Cell Biol 156:233-239. CrossRef Medline

Lee S, Sato Y, Nixon RA (2011) Lysosomal proteolysis inhibition selectively disrupts axonal transport of degradative organelles and causes an Alzheimer's-like axonal dystrophy. J Neurosci 31:7817-7830. CrossRef Medline

Lewis P, Lentz TL (1998) Rabies virus entry into cultured rat hippocampal neurons. J Neurocytol 27:559-573. CrossRef Medline
Maday S, Holzbaur EL (2012a) Autophagosome assembly and cargo capture in the distal axon. Autophagy 8:858-860. CrossRef Medline

Maday S, Wallace KE, Holzbaur EL (2012b) Autophagosomes initiate distally and mature during transport toward the cell soma in primary neurons. J Cell Biol 196:407-417. CrossRef Medline

Mahrhold S, Rummel A, Bigalke H, Davletov B, Binz T (2006) The synaptic vesicle protein $2 \mathrm{C}$ mediates the uptake of botulinum neurotoxin $\mathrm{A}$ into phrenic nerves. FEBS Lett 580:2011-2014. CrossRef Medline

Martin S, Harper CB, May LM, Coulson EJ, Meunier FA, Osborne SL (2013) Inhibition of PIKfyve by YM-201636 dysregulates autophagy and leads to apoptosis-independent neuronal cell death. PLoS One 8:e60152. CrossRef Medline

Matusica D, Skeldal S, Sykes AM, Palstra N, Sharma A, Coulson EJ (2013) An intracellular domain fragment of the p75 neurotrophin receptor (p75(NTR)) enhances tropomyosin receptor kinase A (TrkA) receptor function. J Biol Chem 288:11144-11154. CrossRef Medline

Maucort G, Kasula R, Papadopulos A, Nieminen TA, Rubinsztein-Dunlop H, Meunier FA (2014) Mapping organelle motion reveals a vesicular conveyor belt spatially replenishing secretory vesicles in stimulated chromaffin cells. PLoS One 9:e87242. CrossRef Medline

Meunier FA, Schiavo G, Molgó J (2002) Botulinum neurotoxins: from paralysis to recovery of functional neuromuscular transmission. J Physiol Paris 96:105-113. CrossRef Medline

Meunier FA, Lisk G, Sesardic D, Dolly JO (2003) Dynamics of motor nerve terminal remodeling unveiled using SNARE-cleaving botulinum toxins: the extent and duration are dictated by the sites of SNAP- 25 truncation. Mol Cell Neurosci 22:454-466. CrossRef Medline

Meunier FA, Osborne SL, Hammond GR, Cooke FT, Parker PJ, Domin J, Schiavo G (2005) Phosphatidylinositol 3-kinase C2alpha is essential for ATP-dependent priming of neurosecretory granule exocytosis. Mol Biol Cell 16:4841-4851. CrossRef Medline

Mizushima N, Yamamoto A, Hatano M, Kobayashi Y, Kabeya Y, Suzuki K, Tokuhisa T, Ohsumi Y, Yoshimori T (2001) Dissection of autophagosome formation using Apg5-deficient mouse embryonic stem cells. J Cell Biol 152:657-668. CrossRef Medline

Nair D, Hosy E, Petersen JD, Constals A, Giannone G, Choquet D, Sibarita JB (2013) Super-resolution imaging reveals that AMPA receptors inside synapses are dynamically organized in nanodomains regulated by PSD 95 . J Neurosci 33:13204-13224. CrossRef Medline

Nixon RA (2013) The role of autophagy in neurodegenerative disease. Nat Med 19:983-997. CrossRef Medline

Papadopulos A, Martin S, Tomatis VM, Gormal RS, Meunier FA (2013) Secretagogue stimulation of neurosecretory cells elicits filopodial extensions uncovering new functional release sites. J Neurosci 33:19143-19153. CrossRef Medline

Parton RG, Simons K, Dotti CG (1992) Axonal and dendritic endocytic pathways in cultured neurons. J Cell Biol 119:123-137. Medline

Pellizzari R, Rossetto O, Schiavo G, Montecucco C (1999) Tetanus and botulinum neurotoxins: mechanism of action and therapeutic uses. Philos Trans R Soc Lond B Biol Sci 354:259-268. CrossRef Medline

Pirazzini M, Azarnia Tehran D, Zanetti G, Megighian A, Scorzeto M, Fillo S, Shone CC, Binz T, Rossetto O, Lista F, Montecucco C (2014) Thioredoxin and its reductase are present on synaptic vesicles, and their inhibition prevents the paralysis induced by botulinum neurotoxins. Cell Rep 8:1870-1878. CrossRef Medline

Restani L, Antonucci F, Gianfranceschi L, Rossi C, Rossetto O, Caleo M (2011) Evidence for anterograde transport and transcytosis of botulinum neurotoxin A (BoNT/A). J Neurosci 31:15650-15659. CrossRef Medline

Restani L, Giribaldi F, Manich M, Bercsenyi K, Menendez G, Rossetto O, Caleo M, Schiavo G (2012) Botulinum neurotoxins A and E undergo retrograde axonal transport in primary motor neurons. PLoS Pathog 8:e1003087. CrossRef Medline

Rossetto O, Pirazzini M, Montecucco C (2014) Botulinum neurotoxins: genetic, structural and mechanistic insights. Nat Rev Microbiol 12:535-549. CrossRef Medline

Schiavo G, Santucci A, Dasgupta BR, Mehta PP, Jontes J, Benfenati F, Wilson MC, Montecucco C (1993) Botulinum neurotoxins serotypes A and E cleave SNAP-25 at distinct COOH-terminal peptide bonds. FEBS Lett 335:99-103. CrossRef Medline

Schiavo G, Matteoli M, Montecucco C (2000) Neurotoxins affecting neuroexocytosis. Physiol Rev 80:717-766. Medline

Shehata M, Matsumura H, Okubo-Suzuki R, Ohkawa N, Inokuchi K (2012) 
Neuronal stimulation induces autophagy in hippocampal neurons that is involved in AMPA receptor degradation after chemical long-term depression. J Neurosci 32:10413-10422. CrossRef Medline

Steyer JA, Almers W (1999) Tracking single secretory granules in live chromaffin cells by evanescent-field fluorescence microscopy. Biophys J 76: 2262-2271. CrossRef Medline

Tanida I, Ueno T, Kominami E (2008) LC3 and autophagy. Methods Mol Biol 445:77-88. CrossRef Medline

Taylor AM, Blurton-Jones M, Rhee SW, Cribbs DH, Cotman CW, Jeon NL (2005) A microfluidic culture platform for CNS axonal injury, regeneration and transport. Nat Methods 2:599-605. CrossRef Medline

Verderio C, Grumelli C, Raiteri L, Coco S, Paluzzi S, Caccin P, Rossetto O, Bonanno G, Montecucco C, Matteoli M (2007) Traffic of botulinum toxins A and E in excitatory and inhibitory neurons. Traffic 8:142-153. CrossRef Medline

von Bartheld CS (2004) Axonal transport and neuronal transcytosis of trophic factors, tracers, and pathogens. J Neurobiol 58:295-314. CrossRef Medline

Wang QJ, Ding Y, Kohtz S, Mizushima N, Cristea IM, Rout MP, Chait BT, Zhong Y, Heintz N, Yue Z (2006) Induction of autophagy in axonal dystrophy and degeneration. J Neurosci 26:8057-8068. CrossRef Medline

Wang T, Liu Y, Xu XH, Deng CY, Wu KY, Zhu J, Fu XQ, He M, Luo ZG (2011) Lgl1 activation of rab10 promotes axonal membrane trafficking underlying neuronal polarization. Dev Cell 21:431-444. CrossRef Medline

Wasano K, Hirakawa Y (1994) Lamellar bodies of rat alveolar type 2 cells have late endosomal marker proteins on their limiting membranes. Histochemistry 102:329-335. CrossRef Medline

Weidberg H, Shvets E, Elazar Z (2011) Biogenesis and cargo selectivity of autophagosomes. Annu Rev Biochem 80:125-156. CrossRef Medline

Wen PJ, Osborne SL, Morrow IC, Parton RG, Domin J, Meunier FA (2008) $\mathrm{Ca} 2+$-regulated pool of phosphatidylinositol-3-phosphate produced by phosphatidylinositol 3-kinase C2alpha on neurosecretory vesicles. Mol Biol Cell 19:5593-5603. CrossRef Medline

Wen PJ, Osborne SL, Zanin M, Low PC, Wang HT, Schoenwaelder SM, Jackson SP, Wedlich-Söldner R, Vanhaesebroeck B, Keating DJ, Meunier FA (2011) Phosphatidylinositol(4,5)bisphosphate coordinates actinmediated mobilization and translocation of secretory vesicles to the plasma membrane of chromaffin cells. Nat Commun 2:491. CrossRef Medline

Xie Z, Klionsky DJ (2007) Autophagosome formation: core machinery and adaptations. Nat Cell Biol 9:1102-1109. CrossRef Medline

Yamamoto A, Tagawa Y, Yoshimori T, Moriyama Y, Masaki R, Tashiro Y (1998) Bafilomycin A1 prevents maturation of autophagic vacuoles by inhibiting fusion between autophagosomes and lysosomes in rat hepatoma cell line, H-4-II-E cells. Cell Struct Funct 23:33-42. CrossRef Medline

Yang Y, Coleman M, Zhang L, Zheng X, Yue Z (2013) Autophagy in axonal and dendritic degeneration. Trends Neurosci 36:418-428. CrossRef Medline

Zweifel LS, Kuruvilla R, Ginty DD (2005) Functions and mechanisms of retrograde neurotrophin signalling. Nat Rev Neurosci 6:615-625. CrossRef Medline 